\title{
Brain and Breast Cancer Cells with PTEN Loss of Function Demonstrate Enhanced Durotaxis and RHOB Dependent Amoeboid Migration Using 3D Printed Scaffolds and Aligned Microfiber Tracts
}

\section{Annalena Wieland}

University of Erlangen-Nuernberg: Friedrich-Alexander-Universitat Erlangen-Nurnberg

\section{Pamela L. Strissel}

University of Erlangen-Nuernberg: Friedrich-Alexander-Universitat Erlangen-Nurnberg

Hannah Schorle

University of Erlangen-Nuernberg: Friedrich-Alexander-Universitat Erlangen-Nurnberg

\section{Ezgi Bakirci}

University of Wurzburg: Julius-Maximilians-Universitat Wurzburg

\section{Dieter Janzen}

University of Würzburg: Julius-Maximilians-Universitat Wurzburg

\section{Matthias W. Beckmann}

University of Erlangen-Nuernberg: Friedrich-Alexander-Universitat Erlangen-Nurnberg

\section{Markus Eckstein}

University of Erlangen-Nuernberg: Friedrich-Alexander-Universitat Erlangen-Nurnberg

Paul D. Dalton

University of Würzburg: Julius-Maximilians-Universitat Wurzburg

Reiner Strick ( $\nabla$ reiner.strick@uk-erlangen.de)

Erlangen University Hospital: Universitatsklinikum Erlangen https://orcid.org/0000-0002-8141-7099

\section{Research}

Keywords: 3D tumor model, cell migration, brain cancer, breast cancer, PTEN, RHO, ROCK, durotaxis, amoeboid, melt electrowriting

Posted Date: August 31st, 2021

DOl: https://doi.org/10.21203/rs.3.rs-826338/v1

License: (c) (i) This work is licensed under a Creative Commons Attribution 4.0 International License. Read Full License 
Version of Record: A version of this preprint was published at Cancers on October 14th, 2021. See the published version at https://doi.org/10.3390/cancers13205144. 


\section{Abstract}

Background: Glioblastoma multiforme (GBM) and triple-negative breast cancer (TNBC) with PTEN mutations often lead to brain dissemination with very poor patient outcomes. GBM uses axons and vessels as migratory cues to disseminate, however it is not known, if TNBC shares the same behavior. There is a need to understand brain tumor cell spreading and if GBM and TNBC have similar migration properties involving the signaling pathway RHOB-ROCK-PTEN. We tested for durotaxis, adherence and migration of GBM and TNBC using live-cell imaging and performed molecular analyses on threedimensional (3D) structures.

Methods: Aligned 3D printed scaffolds and microfibers were designed to mimic brain axon tracts and vessels for migration. GBM and TNBC cell lines, each with opposing PTEN genotypes, were analysed with RHO, ROCK and PTEN inhibitors and rescuing PTEN function using live-cell imaging. RNA-sequencing and qPCR of tumor cells in 3D with microfibers were performed, while SEM, confocal microscopy and cell tracking addressed cell morphology.

Results: GBM and TNBC with homozygote PTEN loss of function and RHOB high expression were amoeboid shaped and demonstrated enhanced durotaxis, adhesion and migration on 3D microfibers, in contrast to PTEN wildtype GBM and TNBC showing elongated cells and low RHOB. RNA-sequencing exhibited that RHOB was significantly the highest expressed gene in GBM PTEN loss of function cells. Pathway inhibitors and PTEN rescue of function verified an essential role of RHOB-ROCK-PTEN signaling for durotaxis, adhesion, migration, cell morphology and plasticity using 3D printed microfibers.

Conclusions: This study validates a significant role of a PTEN genotype for cellular properties including durotaxis, adhesion and migration. GBM and TNBC cells with PTEN loss of function have a greater affinity for stiffer brain structures promoting metastasis. We propose the significance of PTEN and RHOB in cellular oncology not only for primary tumors, but also for metastasizing tumors, where RHOB inhibitors could play an essential role for improved therapy.

\section{Background}

Cell migration and invasion are hallmarks of development and cancer [1]. For the ability of cells to migrate, dynamic and spatially regulated changes of the cytoskeleton, cell adhesion and cell interactions with the extracellular matrix (ECM) must occur [2]. Depending upon the cell type, two main migratory phenotypes can be differentiated: 1) single cells, which are amoeboid or mesenchymal; and 2) multicellular or so-called collective cell migration [3]. Cell-cell as well as cell-ECM interactions are crucial for a cell to instantly respond to the environment and further propagate signals, which control cell shape and motility [4]. Specific tissue types harbor different ratios of ECM components, like collagen, laminin, fibronectin and hyaluronic acid [5]. Especially in the brain, higher amounts of laminin and hyaluronic acid are found, where they contribute to normal brain function and tumor stiffness regulating tumor cell motility $[6,7]$. Brain tumor cells sense ECM structures as migratory cues, where they become polarized by 
reorganizing the actin cytoskeleton to facilitate both a protrusive leading and a contractile trailing edge [8]. Cellular protrusions with organized actin filaments, include lamellipodia, filopodia, invadopodia and blebs, which are regulated by various cytoskeletal and signaling pathways [9].

Glioblastoma multiforme (GBM) arise from glial cells of the central nervous system and represent the most aggressive type of human brain tumors [10-12]. Due to the diffuse infiltration of GBM throughout the brain, the inability to achieve complete surgical tumor resection leads to poor patient prognosis with a mean survival of about 15 months [12]. Although, circulating GBM cells have been detected within the blood in $20 \%$ of patients at primary diagnosis, extracranial metastases of GBM are rarely found most likely due to short patient survival [10]. Therefore, it is crucial to obtain a better understanding of GBM migration at a cellular and molecular level.

It is known that GBM cells are attracted to white matter tracts composed of myelinated axons organized into bundles, but also to perivascular spaces of blood vessels where GBM cells migrate and metastasize surrounding brain regions $[11,13,14]$. One explanation for GBM cell attraction could relate to the high mechanical rigidity of axons and blood vessels. This type of physical attraction towards a more rigid environment where cells migrate to and "home in" is defined as durotaxis or mechanical substrate compliance [7, 15]. Other environmental cues influencing cell migration are the following [16]. Chemotaxis, where cells migrate towards diffusible chemical gradients; haptotaxis representing cell movement towards chemical cues on a surface, like ECM proteins, and lastly contact guidance, also called topotaxis or ratchetaxis, where cells use ultrastructures as a guiding track for migration. For example, nanostructures which translate topographical signals to cells influencing directional migration $[17,18]$.

Distant metastasis of primary tumors are known, especially triple negative breast cancer (TNBC), which has a brain metastasis incidence rate of $46 \%[19,20]$. TNBC is a highly malignant and fast progressing subtype of breast cancer, which is diagnostically negative for the expression of the estrogen and progesterone receptors (ER / PR) with no overexpression of the HER2 receptor. Research is ongoing how metastasis of TNBC cells to the brain occurs, but the mechanism how these cells disseminate throughout the brain is still unanswered.

At the molecular level a key player involved in regulating cellular migration and invasion is the Phosphatase and Tensin Homolog (PTEN) protein. PTEN is a known tumor-suppressor antagonizing the oncogenic phosphoinositide 3-kinase (PI3K) pathway, where it dephosphorylates the metabolite phosphatidylinositol-3,4,5-triphosphate (PIP3) to phosphatidylinositol-4,5-bisphosphate (PIP2) inhibiting downstream signaling. PTEN loss of function results in constitutively active PI3K signaling and induces proliferation, migration as well as cell survival [21, 22]. PTEN mutations are one of the most common genetic alterations in GBM and are directly associated with malignant transformation and metastasis, but also noted in up to $40 \%$ of primary breast cancers associated with therapeutic resistance and a shorter patient overall survival [23-27]. Interestingly, PI3K activation or loss of PTEN was found in $77 \%$ of brain metastases or $25-71 \%$ of breast tumors metastasized to the brain, respectively [28-30]. 
Other essential factors for cell migration are the ras homolog family member (RHO) GTPases, which include 20 mammalian genes, play a more determining role regulating actin polymerization, depolymerization and activity of actin-associated myosins during migration [31]. PTEN is required for directional movement of cells via different cellular localizations and activation through RHO GTPase and Rho associated coiled-coil containing protein kinase (ROCK) signaling [32]. Besides the main RHO GTPase RHOA, RHOB plays an essential role for actin cytoskeleton and especially for membrane blebbing and amoeboid migration [33].

Biomedical materials developed for use in tissue engineering are essential for modeling 3D tumor growth, migration and invasion $[34,35]$. A relatively new 3D printing technology, melt electrowriting (MEW) has been used to fabricate complex multi-scaled architectural biomaterial structures [36, 37] from microfibers [38] also used for clinical purposes. MEW processes polymers, which can be implemented within a biological context for 3D cell cultures recapitulating aspects of the in vivo environment are novel tools for $3 \mathrm{D}$ tumor cell modeling [39-41].

In this investigation two different 3D MEW poly-( $\varepsilon$-caprolactone) (PCL) structural designs were used as topographical guides to mimic axons and blood vessels in the brain to assess, if GBM and TNBC cells have similar cellular activities. RNA sequencing (RNA-seq) was used to shed light on the gene pathways regulating adhesion, migration and cell morphology. Our main findings demonstrate that GBM and TNBC types have enhanced durotaxis and topotaxis and increased migration influenced by the PCL MEW fibers. We show that enhancement of these cellular activities stems from mutated PTEN variants with loss of function and de-regulated RHOB signaling. This study validates a significant role for the PTEN loss of function genotype for these cellular processes and explains why these tumor cells have a greater affinity for stiffer structures, like axons and blood vessels promoting dissemination throughout the brain.

\section{Methods}

\section{Cell lines}

All cell lines used in this investigation were purchased from the American Type Culture Collection (ATCC, Rockville MD, USA) and tested regularly for mycoplasma infection according to manufacturer's instructions (Minerva Biolabs, Berlin, Germany). GBM cell lines U87 MG (U87 GBM) (ATCC $®$ HTB 14 $4^{\text {TM }}$ ) and LN18 (LN18 GBM) (ATCC ${ }^{8}$ CRL-2610 ${ }^{\mathrm{TM}}$ ) and the triple negative breast cancer (TNBC) cell lines MDA MB 231 (ATCC $\AA$ HTB26 ${ }^{\text {TM }}$ ) and MDA MB 468 (ATCC $\AA$ HTB132 $2^{\text {TM }}$ ) were grown and maintained as described below. U87 GBM cells were cultured in Minimum Essential Medium (MEM); LN18 GBM and HTB26 were cultured in Dulbecco's modified Eagle's medium (DMEM) and HTB132 in Dulbecco's Modified Eagle Medium: Nutrient Mixture F-12 (DMEM/F12). All media were supplemented with $10 \%$ fetal bovine serum (FBS), 2 mM L-Glutamine, 10 mM HEPES Buffer and 1 x Non-essential Amino Acids (all media, FBS, and supplements from Gibco ${ }^{\mathrm{TM}}$ Thermo Fisher Scientific, Waltham, MA). Cells were maintained in 75 $\mathrm{cm}$ tissue culture flasks in a humid atmosphere with $5 \% \mathrm{CO}_{2}$ at $37^{\circ} \mathrm{C}$. Cells were passaged at $70-80 \%$ confluency using 0.0625 \% Trypsin. Additional information regarding the cell lines: the U87 GBM cell line 
has a PTEN gene splice donor mutation of exon three resulting in an in-frame exon three deletion and PTEN loss of function; whereas LN18 GBM is PTEN wt [42]. The TNBC HTB132 cell line has a PTEN gene splice donor mutation of exon four resulting in an in-frame deletion of exon four and PTEN loss of function $[42,43]$. The HTB26 cell line is PTEN wt. For simplicity both U87 GBM and HTB132 cell lines will be referred to as PTEN loss of function; whereas LN18 GBM and HTB26 as PTEN wt.

\section{MEW printing and glass slide treatment}

A custom built MEW printerwas used to fabricate box-pore scaffolds as well as aligned and stacked microfiber tracts as previously described [36, 44]. Briefly, MEW was performed using medicalgrade PCL (PURASORB PC 12, Lot\#1712002224, 05/2018, Corbion Inc, Amsterdam, Netherlands) at $21 \pm$ $3{ }^{\circ} \mathrm{C}$ and a humidity of $40 \pm 5 \%$. The following parameters were used for MEW processing; scaffolds were printed at $85^{\circ} \mathrm{C}, 3 \mathrm{bar}, 25 \mathrm{G}$ nozzle, $4 \mathrm{~mm}$ collector distance and $6 \mathrm{kV}$ voltage applied. A $48 \times 96 \mathrm{~mm}$ rectangular mesh was direct-written and cut to $9 \mathrm{~mm}$ disks with an infrared laser. Aligned microfiber tracts were printed at $85^{\circ} \mathrm{C} ; 0.5$ bar of air pressure; $22 \mathrm{G}$ nozzle and $6.5 \mathrm{kV}$ voltage applied across a $3.85 \mathrm{~mm}$ collector distance. The interfiber distance was set at $200 \mu \mathrm{m}$ with 10 layers in G-code for both scaffolds. Scaffolds and aligned microfibers were printed onto a metal surface or glass coverslips, respectively, where the glass surface was previously coated with NCO-terminated -star-shaped poly(ethylene oxide-stat-propylene oxide) (sP(EO-stat-PO) (provided by DWI Leibnitz Institute for Interactive Materials, Aachen, Germany) in order to facilitate scaffold adherence but decrease surface protein adsorption. Prior to SP(EO-stat-PO coating, coverslips were washed with acetone, water, isopropanol and dried using an air pressure gun, treated with $100 \%$ oxygen plasma in a plasma generator (Pico low-pressure plasma system, Diener Electronic GmbH, Ebhausen, Germany) then incubated in a desiccator with 3-aminopropyl-trimethoxysilane for surface activation. Coverslips were then homogenously rotationally spun and coated at $2500 \mathrm{rpm}$ for $40 \mathrm{~s}$ with $10 \mathrm{mg} \mathrm{ml}^{-1} \mathrm{NCO}$-sP(EO-statPO) in $10 \%$ (v/v) tetrahydrofuran (Merck, Darmstadt, Germany) - MilliQ water. Coated coverslips were ready for MEW printing within $24 \mathrm{~h}$.

\section{MEW scaffold functionalization}

Scaffolds were placed into a $15.6 \mathrm{~mm}$ culture dish (24-well plate) (Waltham, Massachusetts, USA) or aligned microfiber tracts placed into a $34.8 \mathrm{~mm}$ culture dish (6-well plate) (Corning, Corning, New York, USA) then sterilized with UV-light for $30 \mathrm{~min}$, incubated with $1 \mathrm{M} \mathrm{NaOH}$ for $10 \mathrm{~min}$ and finally washed 5 times with $1 \times$ PBS. For functionalization, scaffolds or aligned microfiber tracts were incubated with $10 \mu \mathrm{g}$ $\mathrm{ml}^{-1}$ laminin-111 (BioLamina, Sundbyberg, Sweden) at $4{ }^{\circ} \mathrm{C}$ overnight.

\section{U87 GBM stably expressing the farnesylated-tdTomato fluorescent protein}

A stable transfected U87 GBM cell line was established expressing the farnesylated-tdTomato fluorescent protein in order to track the 3D movement of cells within Matrigel with or without scaffolds (cell network formation assay see below). For cloning of the dT-farnesyl-vector, the cDNA of the farnesylated-tdTomato fluorescent protein was reversed transcribed from the tdTomato-farnesyl-5 gene (Addgene, Watertown, 
MA, USA) implementing primers containing a Bam $\mathrm{HI}$ and Notl restriction enzyme sites in order to replace the AcGFP cDNA in the pLVX-AcGFP-N1 vector (Clontech, Mountain View, CA, USA). Following ligation into the pLVX-AcGFP-N1 vector, bacterial transformation, purified plasmid DNA was then expanded using the NucleoBond Xtra Maxi Kit (Macherey-Nagel, Düren, Germany) and verified for the correct tdTomatofarnesyl-5 gene sequence of the construct (Eurofins Genomics Germany $\mathrm{GmbH}$, Ebersberg, Germany). The pLVX-tdTomato-farnesyl-5-N1 vector was then co-transfected with the packaging plasmid psPAX.2 (Addgene, Watertown, MA, USA) and the envelope vector VSV-G (Addgene, Watertown, MA, USA) into LentiX 293 T cells (Takara Bio Inc., Kusatsu, Shiga, Japan) using the Lipofectamine 2000 reagent (Thermo Fisher Scientific, Waltham, MA, USA). $48 \mathrm{~h}$ post transfection, the lentivirus-containing supernatant was harvested and briefly centrifuged, before being concentrated with the LentiX concentrator (Takara Bio Inc., Kusatsu, Shiga, Japan). For subsequent reverse transduction of U87 GBM cells, the concentrated virus (107 infectious units $\mathrm{ml}^{-1}$ ) was dispensed on 100,000 cells per $9.6 \mathrm{~cm}^{2}$. Successfully transduced U87 cells were further selected with $3 \mu \mathrm{m} \mathrm{ml}^{-1}$ Puromycin (Thermo Fisher, Waltham, Masssachussettes, USA).

\section{Cell network formation assay}

In order to perform the cell network formation assay, each cell suspension contained 50,000 U87 GBM farnesylated-tdTomato cells and Matrigel (Corning, Corning, New York, USA) at a final concentration of $4.5 \mathrm{mg} \mathrm{mL}^{-1}$ in a final volume of $150 \mu \mathrm{l}$ and then was pipetted onto a scaffold in a $15.6 \mathrm{~mm}$ culture dish. After $30 \mathrm{~min}$ incubation at $37^{\circ} \mathrm{C}, 100 \mu \mathrm{l}$ culture medium was added. Laminin-coated scaffolds were imaged every $10 \mathrm{~min}$ at $568 \mathrm{~nm}$ for a total of $20 \mathrm{~h}$ using an inverted microscope (Olympus IX83, cellSens Software V1.16, Olympus Corporation, Tokyo, Japan). Six experiments were performed for U87 GBM cells with scaffolds and eight experiments with U87 GBM cells Matrigel alone. The AVI-videos were converted into TIF-files using a Python script. The cell network formation assay was analyzed using the online Tool WimTube (Wimasis, Onimagin Technologies SCA, Spain). We previously implemented the WimTube program to analyze primary breast adipose stem cells, which form and resemble endothelial capillary like structures with Matrigel [41]. In this present study the following structures were quantified using WimTube for the cell network formation assay with U87 GBM cells: 1) the total number of cell loop structures and 2) the total number of branch-like structures.

\section{Cell migration after PTEN, RHO and ROCK inhibition or rescuing PTEN function using live cell imaging}

Live cell imaging was performed using an inverted microscope and phase contrast (Olympus IX83, cellSens Software V1.16, Olympus Corporation, Tokyo, Japan). For each migration experiment, 20,000 cells of a respective cell line were initially seeded onto laminin-coated aligned microfiber tracts and after a 15 min incubation at $37^{\circ} \mathrm{C}, 100 \mu \mathrm{l}$ of culture medium was added. Aligned microfibers were imaged every $10 \mathrm{~min}$ for up to $15 \mathrm{~h}$. The AVI-videos were converted into TIF-files, using a Python script. To inhibit the PTEN wt protein, LN18 GBM cells $\left(300,000\right.$ per $\left.9.6 \mathrm{~cm}^{2}\right)$ were treated with the PTEN inhibitor SF-1670 (PTENi) (Sellekchem, Houston, Texas, USA) at a concentration of $3 \mu \mathrm{M}$ starting $48 \mathrm{~h}$ prior to migration live cell imaging ( $n=7$ experiments). To rescue U87 GBM cells with a PTEN loss of function, we cloned the 
human PTEN wt gene under control of CMV in the overexpressing pRK5 vector. PTEN cDNA was reversed transcribed from human whole blood RNA using primers containing an EcoR1 and EcoR1/Sal1 sites. The PTEN PCR product (1230 bp) was then ligated into the pRK5 Vector (pRK5-PTEN) and confirmed by DNA sequencing. U87 GBM cells with a PTEN loss of function were transfected with $3 \mu \mathrm{g}$ pRK5-PTEN vector using JetPEI® according to manufacturer's instructions (VWR, Radnor, Pennsylvania, USA). The U87 GBM PTEN transfected cells were assayed for migration on laminin-coated aligned microfibers using live cell imaging after $48 \mathrm{~h}$ ( $\mathrm{n}=4$ experiments for control; $\mathrm{n}=3$ experiments for transfection). To inhibit the RHOassociated, coiled-coil containing protein kinase (ROCK), we treated U87 GBM cells (350,000 per 9.6 $\mathrm{cm}^{2}$ ) with the ROCK-inhibitor Y-27632 (ROCKi) (Stemcell Technologies, Vancouver, Canada) at a concentration of $5 \mu \mathrm{M}$, starting $24 \mathrm{~h}$ prior to the migration assay using live cell imaging ) ( $\mathrm{n}=7$ control and $\mathrm{n}=9$ ROCKi experiments). For RHO inhibition of respective cell lines $\left(350,000\right.$ per $\left.9.6 \mathrm{~cm}^{2}\right), 3 \mu \mathrm{g} / \mathrm{ml}$ of the $\mathrm{RHO}$ Inhibitor (C3 Trans based) (RHOi) (Biozol, Eching, Germany) was added to culture media containing $5 \% \mathrm{FCS}$, starting $6 \mathrm{~h}$ prior to the migration assay live-cell imaging. Cells were then incubated with laminin-coated aligned microfiber tracts and imaged every $10 \mathrm{~min}$ for a total of $20 \mathrm{~h}$ (U87 GBM PTEN loss of function: $n=4$ experiments, HTB132 PTEN loss of function: $n=3$ experiments). The AVI-videos were converted into TIF files using a Python script. The migration speed in $\mu \mathrm{m} / \mathrm{h}$ was calculated using the manual tracking tool of ImageJ/Fiji.

\section{Cell Viability}

Cell viability after treating cell lines with the RHOi was assessed using a Live/Dead assay. In brief, cells were incubated for $30 \mathrm{~min}$ at $21^{\circ} \mathrm{C}$ for $30 \mathrm{~min}$ with $2 \times 10^{-6} \mathrm{M}$ Calcein-AM (green/living cells; Thermo Fisher Scientific, Waltham, MA, USA) and $2 \times 10^{-6} \mathrm{M}$ ethidium homodimer I (red/dead cells, Sigma-Aldrich, St. Louis, MO, USA) in 1x PBS.

\section{Immunocytochemistry and F-actin staining}

U87 GBM cells $\left(50,000\right.$ cells grown in $4.5 \mathrm{mg} \mathrm{mL}^{-1}$ Matrigel (Corning, NY, USA) with scaffolds were stained for $ß$-Tubulin and microtubule-associated protein 2 (MAP2) to assess 3D cell growth and network formation at day 1 , day 3 and day 6 . All steps were performed at $21^{\circ} \mathrm{C}$. Cells were fixed for 15 min with 2 $\%$ paraformaldehyde (PFA), washed with 1x PBS, and blocked / permeabilized for 3 min with $5 \%$ goat serum and $0.2 \%$ Triton X-100 in 1x PBS. Cells were incubated with primary antibodies Anti-ß-Tubulin (1: 500, Abcam, Cambridge, UK) and Anti-MAP2 for $2 \mathrm{~h}$ (1: 500, Sigma Aldrich, St. Louis, Missouri, USA). Following washing, cells were incubated for 45 min with secondary Dylight-488 Alexa and Dylight-594 (both 1: 200 dilutions; Sigma Aldrich, St. Louis, Missouri, USA). For F-actin staining, Actin Green 488 ready probes were used (Thermo Fisher Scientific, Waltham, MA, USA). To stain cell nuclei DRAQ5 (BioStatus, Shepshed, UK) or Hoechst 33342 (Thermo Fisher Scientific, Waltham, MA, USA) were used according to manufacturer's instructions. The computer software Imaris was used for 3D reconstructions (Oxford Instruments, Abingdon, UK). Cells were either incubated with Actin Red 555 Ready Probes Reagent ( $2 \mu \mathrm{g} /$ $\mathrm{ml}$ ) (Rhodamine phalloidin) or Actin Green 488 ready probes (both from Thermo Fisher Scientific, 
Waltham, MA, USA) specific for F-actin, and DRAQ5 (Biostatus Ltd., Shepshed, UK) for cell nuclei according to manufacturer's instructions.

\section{Confocal and Scanning Electron Microscopy}

Cells were imaged using a confocal laser scanning microscope (Leica, SP5X, $20 \mathrm{x}$ dip-in water-immersion objective; numerical aperture (NA) 1.0) with laser power set to $1.2 \mathrm{~mW}$ to avoid photobleaching. F-Actin Alexa 488 was detected with an Argon laser at 488 nm; F-Actin Red 555 (Tetramethylrhodamine (TRITC)) with an Argon laser at $540 \mathrm{~nm}$, and DRAQ5 with a He-Ne laser at $633 \mathrm{~nm}$. Hoechst 33342 was detected with a UV laser at $350 \mathrm{~nm}$. Images of cells with scaffolds represent an overlay of 35-40 z-stack sections. Confocal images of cells on aligned microfibers represent an overlay ranging from 18-21 z-stack sections (each step $0.99 \mu \mathrm{m}$ equaling $17.82 \mu \mathrm{m}-20.79 \mu \mathrm{m}$ with a line average of 3 .

For scanning electron microscopy (SEM), a Zeiss Crossbeam 340 scanning electron microscope (Carl Zeiss Microscopy $\mathrm{GmbH}$, Oberkochen, Germany) was used. Aligned microfibers were washed with PBS and incubated in $6 \%$ glutaraldehyde (Sigma-Aldrich, Schnelldorf, Germany) for 15 min. After a washing step in ice-cold PBS, the samples were dehydrated by increasing concentrations of ethanol from 50 to $100 \%$. The samples were incubated in hexamethyldisilazane (Sigma-Aldrich, Schnelldorf, Germany) for $15 \mathrm{~min}$ and dried overnight. All samples were sputter-coated with a $4 \mathrm{~nm}$ layer of platinum with a Leica EM ACE600 (Leica Microsystems, Wetzlar, Germany) before imaging.

\section{RNA Extraction, qPCR and RNA-sequencing}

For RNA-extraction, 50,000 cells of each representative cell line were grown in Matrigel in the presence of scaffolds. RNA was extracted at day 3 using TriFast ${ }^{\circledR}$ PeqGOLD (PEQLAB Biotechnologie, Erlangen, Germany) according to manufacturer's instructions. All samples were digested using DNase I to further fractionate the RNA and then precipitated in the presence of Glycogen (both from Sigma-Aldrich, St. Louis, Missouri, USA). All RNAs were stored at $-80^{\circ} \mathrm{C}$. RNA was reversed-transcribed into cDNA using the High-Capacity cDNA Reverse Transcription Kit (Thermo Fisher Scientific, Waltham, MA, USA). For quantitative real-time PCR, SYBR Select Master Mix (Life Technologies, 4472919) and MicroAMPFast 96well Reaction-plates (Applied Biosystems, Forster City, CA, USA) were used with a StepOne ${ }^{\text {TM }}$ Real-Time PCR System (Thermo Fisher Scientific, Waltham, MA, USA). All kits were used accordingly to the manufacturer's instructions. Primer Sequences: RPL13-TF (5'-CTGCTGAAGAACTGAAACTGGC-3'), RPL13BR (5'-CTCTTCCTCAGTGATGACTGGA-3'), RHOA-TF (5'-TTCCCAAGAAACTGG-3'), RHOA-BR (5'CATACACCTCTGGGA-3'), RHOB-TF (5'-CATCCAAGCCTACGA-3'), RHOB-BR (5'CAGTTGATGCAGCCGTTCTG-3').

For RNA-sequencing (RNA-seq) $200 \mathrm{ng}$ poly-A purified mRNA was isolated from U87 GBM cells grown in 2D and in 3D with Matrigel and scaffolds from day 3 ( $n=3$ independent experiments were pooled). RNAseq was performed by Eurofins Genomics Germany (Ebersberg, Germany). Paired-end read RNA-seq ( $2 x$ 150 bp) was executed via Illumina with 31,779,803 clean reads for U87 GBM in 2D and 33,331,487 for U87 GBM in 3D and scaffolds. Transcriptome analyses were performed by mapping and quantification of 
transcripts against a reference genome and a pairwise comparison of expression determining significant fold changes.

\section{Western Blot}

Cell lysates were loaded onto Mini-Protean TGX Precast protein gels (BioRad, Hercules, CA, USA) and run at $100 \mathrm{~V}$ for $1 \mathrm{~h}$. Gels were semi-dry transferred onto PVDF Membranes. The membrane was incubated in $1 \mathrm{x}$ PBS $+0.1 \%$ Tween-20 buffer (1x PBST) with $3 \%$ milk powder (blocking buffer) for $1 \mathrm{~h}$ at room temperature. PTEN antibody (Cell Signaling, Danvers, Massachusetts, USA) and GAPDH antibody (Santa Cruz Biotechnologies, Dallas, Texas, USA) were diluted 1: 750 in blocking buffer and incubated overnight at $4{ }^{\circ} \mathrm{C}$. Washes were performed with PBST before the addition of goat-anti-rabbit-HRP (Cell Signaling, Danvers, Massachusetts, USA) (1:500) for $1 \mathrm{~h}$ at room temperature. For protein detection the membrane was incubated for 3 min in HRP juice (PJK GmbH, Kleinbittersdorf, Germany) and visualized using the Amersham $^{\mathrm{TM}}$ Imager 600 (GE Lifesciences, Little Chalfont, UK).

\section{Statistical analysis and Data Presentation}

All statistical analyses were made with GraphPad Prism 8 for Windows (GraphPad Software) using nonparametric statistical tests (Mann-Whitney-Test). Breast and glioblastoma pan cancer atlas, The Cancer Genome Atlas (TCGA) data sets were accessed via cbioportal [45]. Categorized mutational data for PTEN (PTEN mutation = pathogenic and likely pathogenic mutations; $P T E N$ splice variants and $\mathrm{wt}$ ) and $R H O B / R H O A$ gene expression were downloaded and correlated to each other. For statistical testing nonparametric Mann-Whitney tests were used.

\section{Results}

\section{Design and fabrication of scaffolds as well as 3D aligned microfibers for cellular attraction, adhesion and migration properties.}

Previously we demonstrated that 8-chamber radial structures using MEW and filled with different concentrations of 3D matrices was an important tool to determine the optimal matrix and concentration for growth of the U87 GBM human cell line [46]. In a different study, aligned solution electrospun nanofiber sheets enhanced migration of U87 GBM cells, xenographed in mice, away from the primary brain tumor site to an extracortical site [47]. Thus, tailoring MEW printed fiber structural designs is helpful to address biological functions of tumor cells. Here we designed MEW 3D box-pore scaffolds or aligned microfibers in order to resemble axons and blood vessel tracts (Fig. 1). SEM measurements for both scaffolds and aligned microfibers consisted of 10 layers of single aligned PCL microfibers with an average fiber diameter of $10 \mu \mathrm{m}$, an inter-fiber bundle distance of $200 \mu \mathrm{m}$ and an overall height of approximately $100 \mu \mathrm{m}$ (Fig. 1B, C). Additionally, for scaffolds, ten layers of microfibers were deposited in each direction $\left(0^{\circ}\right.$ and $\left.90^{\circ}\right)$ resulting in a 20 -fiber overlap at intersections (Fig. 1B). 
Since GBM and breast tumor cells can disseminate throughout the brain via axons and blood vessels, we first tested, if U87 GBM cells have an affinity for the scaffolds. Growing U87 GBM cells in 3D with Matrigel ( $\left.4.5 \mathrm{mg} \mathrm{mL}^{-1}\right)$ and laminin-coated scaffolds, resulted in an increased cellular affinity for the scaffold within $24 \mathrm{~h}$ and continued until $72 \mathrm{~h}$ (Figure S1). We observed at 24 to $72 \mathrm{~h}$ distinct amoeboid cells, migrating towards and associated with the scaffold (Figure S1A). By day six, U87 GBM cells became more extended, where they formed distinct cellular networks between the scaffolds, but were also wrapped around aligned microfibers (Fig. 2A, Figure S1A). In order to directly test an affinity of GBM cells for scaffolds, we live-imaged and tracked U87-td-farnesyl expressing GBM cells, which emit a red cytosolic fluorescence, in a time kinetic manner up to $18 \mathrm{~h}$ in Matrigel and laminin-coated scaffolds or Matrigel alone (Fig. 2B, Video S1, Video S2). Only in the presence of scaffolds, tumor cells actively migrated toward and adhered to the scaffold forming loop and branch-like cellular networks (Fig. 2B, C). Cell attraction appeared to occur equally along aligned microfibers and at the corners of scaffold structures, thus showing no topographical preference for a particular scaffold region. The total number of U87 GBM cellular loops and branches in the presence of the scaffold significantly peaked at $10 \mathrm{~h}$ and 15 h compared to Matrigel alone (Fig. 2 C).

Previously it was shown that the stiffness of Matrigel (at $4.5 \mathrm{mg} / \mathrm{ml}$ ) represents a soft matrix of $<100 \mathrm{~Pa}$ (Young's modulus), but scaffolds exhibited a high stiffness of $\sim 15 \mathrm{kPa}[39,40]$. A combination of hydrogel with scaffolds positively influences the overall handling by increasing stiffness synergistically $[39,40]$. In general, a higher matrix rigidity activates durotaxis directed cell migration toward stiffer substrates $[48,49]$. We interpret our findings that a durotactic tumor cell response toward a substrate of a higher stiffness such as scaffolds with aligned microfibers represent a strong physical cue (Fig. 1, 2). However, our findings demonstrating the wrapping of U87 GBM cells around MEW fibers also suggest that the fiber topography affects cell behavior (Fig. 2A).

\section{GBM and breast cancer cell migratory behavior in the brain using 3D aligned microfibers}

Following our findings demonstrating a strong durotactic response of U87 GBM cells for scaffolds, we next implemented aligned microfibers (Fig. 1C) to analyze the cell migratory behaviour of GBM and TNBC cell lines in detail using live cell imaging (Fig. 3, 4). We first tested U87 GBM cell migration on aligned microfibers coated with laminin-111 (Fig. 3, Figure S1B) as laminin-111 is the most prominent brain ECM subtype and also enriched on blood vessels $[50,51]$. Similar to scaffolds, an enhanced durotactic response of U87 GBM cells was also observed for aligned microfibers as well as active cell migration (Fig. 3A, Video S3). Furthermore, SEM indicated that most U87 GBM cells were morphologically round and organized onto single microfibers supporting a topographical cue for movement (Fig. 3B).

In striking contrast to U87 GBM cells, LN18 GBM cells showed no active durotactic response for laminincoated aligned microfibers and no migration (Fig. 4A, Video S4). However, over a $10 \mathrm{~h}$ period of time some cells could adhere to aligned microfibers, but did not migrate. We hypothesized that these differences between U87 GBM and LN18 GBM cells could be due to their opposite PTEN genotypes (U87 GBMPTEN loss of function with no PTEN protein expression; LN18 GBM PTEN wt) (Figure S2A,B). Based 
upon the known regulatory role of PTEN in migration, we performed live cell imaging experiments modulating PTEN function. Transfecting and rescuing U87 GBM cells (PTEN loss of function) with a PTEN wt overexpressing vector (Figure S2B) significantly blunted migration speed from $32.4 \mu \mathrm{m} / \mathrm{h}$ to $29.5 \mu \mathrm{m} / \mathrm{h}(\mathrm{p}=0.0341)$ (Fig. 4C). In contrast, treatment of LN18 GBM cells (PTEN wt) with a PTEN inhibitor (PTENi) restored a durotactic response, adherence and migration on laminin-coated aligned microfibers (Fig. 4A, Video S5). A highly significant cell migration speed of $16.4 \mu \mathrm{m} / \mathrm{h}$ compared to no migration without inhibitor treatment $(p=0.0002)$ was found (Fig. 4D).

We next tested, if two TNBC cell lines with opposing PTEN genotypes behaved similar to GBM cells for durotaxis, cell adhesion and migration using the same experimental conditions. Results showed that HTB132 cells (PTEN loss of function) and HTB26 cells (PTEN wt) were comparable to GBM cell lines, but with some differences (Fig. 4B, E). For example, HTB132 cells (PTEN loss of function) like U87 GBM cells demonstrated a strong durotactic response for aligned microfibers, cell adhesion and migration (Video S6). Although both PTEN wt, in contrast to LN18 GBM, HTB26 cells showed a more collective cell adhesion, but migration only occurred as single cells (Video S7). However, HTB26 migrated significantly slower at $17 \mu \mathrm{m} / \mathrm{h}$ on aligned microfibers than HTB132 (PTEN loss of function) at $26.5 \mu \mathrm{m} / \mathrm{h}$ ( $\mathrm{p}<$ 0.0001) (Fig. 4B, E).

\section{RHOB as a novel regulator of migration}

To further explain PTEN regulation of GBM and TNBC cell migration, we focused on the RHO-ROCK signaling pathway (Fig. 5B). Next to RHOB and RHOC, RHOA has been described as the most prominent regulator of the migration signaling pathway. ${ }^{[51]}$ To gain insight into the genes including RHO-ROCK signaling and other pathways, we performed RNA-seq from U87 GBM cells grown in 3D with Matrigel and scaffolds compared to 2D controls. Results showed a highly significant 46.15-fold increase of RHOB expression in 3D versus 2D ( $p=0.0001)$ (Fig. 5A, Supplemental Table 1). Differential gene expression of RHOB signaling members as well as actin and myosin genes support regulation of migration (Fig. $5 \mathrm{C}$ ). On the other hand, genes involved in other migration modes, like neuronal migration via nucleokinesis (nuclear piston migration) and astrocyte migration (astrogliosis) were not increased, supporting the hypothesis that GBM cell migration is predominantly regulated by RHO/ROCK signaling (Fig. 5C) [52].

The significant role of RHOB was validated using quantitative real time PCR (qPCR) for both U87 GBM and HTB132 cells using the same 3D and 2D culture conditions (Fig. 5D). Furthermore, RHOB expression was significantly higher than RHOA for U87 GBM cells in 3D. In contrast, both PTEN wt LN18 GBM and HTB26 cells showed no significant increase of RHOB gene expression compared to 2D (Figure S2C). Our results support that a PTEN loss of function is linked with higher RHOB expression and regulation of migration.

Based upon our RNA-Seq and qPCR results we initiated functional cell culture studies to further unravel the role of RHOB and pathway members in the migratory behavior of U87 GBM and HTB132 cells (Fig. 5B). Treatment of U87 GBM cells with a ROCKi demonstrated a highly significant decrease of migration 
speed to $24.5 \mu \mathrm{m} / \mathrm{h}(\mathrm{p}<0.0001)$ on aligned microfibers compared to control, where cells migrated at an average speed of $31.5 \mu \mathrm{m} / \mathrm{h}$ (Fig. 6A, B). Further in line with RHO/ROCK signaling, treating both PTEN loss of function U87 GBM and HTB132 cells with a RHO inhibitor (RHOi) blunted durotaxis and adhesion, but completely inhibited cell migration, while both cell types remained viable (Fig. 6C, Video S8). This result supports that RHOi primarily inhibits cell migration, which also is necessary for the durotactic response.

Finally, we asked the question, if there was a clinical correlation between primary GBM and breast cancers with PTEN wt or PTEN splice or other mutations and RHOB expression using The Cancer Genome Atlas (TCGA). Although rare, primary GBM and breast tumors with PTEN splice mutations had significantly higher RHOB gene expression versus tumors with PTEN wt or other PTEN mutations (Fig. 7A). Upon further analyses 7 of 10 of the primary GBM and breast cancer tumors with PTEN splice mutations and high RHOB expression a homozygote PTEN loss of function was found. In line with TCGA primary tumors both U87 GBM and HTB132 cells harbor PTEN splice variants leading to a homozygote PTEN loss of function (Figure S2A for U87 GBM). In contrast, analyzing RHOA expression in primary GBM and breast tumors, using the same TCGA cohorts, showed no significant RHOA expression for tumors with PTEN splice variants however, a significant higher expression of RHOA ( 10\%) was found in tumors with PTEN mutations versus tumors with PTEN wt $(\mathrm{p}=0.001)$. The above findings along with our cell culture studies support a prominent role for RHOB and PTEN splice variants with PTEN loss of function in regulating tumor cell migration of GBM and breast cancers.

\section{RHOB signaling as a regulator of cell morphology}

A more in-depth view of RHOB signaling as regulated by PTEN supports a cellular decision of either an amoeboid or mesenchymal cell shape as well as movement (Fig. 5B). Microscopic analyses comparing both GBM and TNBC cell lines with opposing PTEN genotypes on laminin-coated aligned microfibers noted significant differences in cell morphology. For example, SEM demonstrated that high RHOB expressing U87 GBM and HTB132 cellswith PTEN loss of function were amoeboid and primarily located mainly on single fibers (Fig. 7B). In contrast, both low RHOB expressing and PTEN wt LN18 GBM and HTB26 cells were flat and spread over 2-3 fibers. Measuring the cell heights confirmed that amoeboid U87 GBM and HTB132 cells had an average cell height of $9.9 \mu \mathrm{m}$ and $12.2 \mu \mathrm{m}$, respectively (Fig. 7D). These values were significantly higher compared to HTB26 PTEN wt cells and U87 GBM cells transfected with a PTEN wt overexpressing vector, which had an average cell height of $8.3 \mu \mathrm{m}$ and $6.5 \mu \mathrm{m}$, respectively.

Using confocal laser microscopy detecting F-actin confirmed cell morphologies as observed by SEM (Fig. 7B, C). Congruent with an amoeboid morphology, U87 GBM cells and HTB132 cells showed distinct Factin protrusions with different sizes at the leading edge, but also small actin blebs on the surface of HTB132 cells (Fig. 7C, 8C and 8G with white arrows). A prominent F-actin (Phalloidin positive) staining was also noted at the cell surface for both cell lines along the membrane with different intensities (Fig. 
$7 \mathrm{C}, 8 \mathrm{C}$ and $8 \mathrm{G}$ with red arrows). The nucleus of the U87 GBM cells was primarily located at the rear of the cell, opposite to the direction of movement (Fig. 7C, 8C).

\section{Skating snail-like cell migration and motor clutch model}

To unravel a more detailed analysis of the migration mode, we focused on U87 GBM and HTB132 tumor cells with PTEN loss of function and high RHOB expression. Using live cell imaging with a higher magnification we compared cell morphology, plasticity modes, the total migratory distance and persistence behavior on aligned microfibers. Distinct amoeboid plasticity modes resembling "snail-like" crawling followed by alternating fast movements was common for both tumor cell types (Fig. 7C, 8A-G, Figure S3, Video S9, S10). These skating snail-like cellular forms resemble how speed skaters on ice initially propel their forces forward to accelerate and then skate in a gliding manner with higher speed.

For example, one mode showed that skating snail-like U87 GBM cells were initially slow, then accelerated (between 9 - $15 \mathrm{~min}$ ) and slowed down again (between 30 - $42 \mathrm{~min}$ ) (Fig. 8A, D, G top, Figure S3B). Another skating snail-like U87 GBM cell mode demonstrated an instant acceleration to a fast mode then slowed down and stopped (Fig. 8E, G bottom; Figure S3B). Lastly, a skating snail-like U87 GBM cell mode showed a slow "crawling" and then a faster sliding with long cellular protrusions at the leading edge (Fig. 8B, C, F, Figure S3B). These changes in cellular plasticity modes were dramatically pointing to an intrinsic nature of these tumor cells. However, when cells paused their migration on aligned microfibers, both U87 GBM and HTB132 cells displayed a more elongated and flattened morphology (Fig. 8A,B,D,E,F, Figure S3A, $B)$. Analyzing the cell surface length along aligned microfibers of moving cells over time demonstrated that cells during fast movements had the shortest length compared to slow and halted cells (Fig. $8 \mathrm{H}$ ). Next, we addressed the total migratory distance and persistent migration of U87 GBM and HTB132 cells. Comparing the total time that cells migrated on aligned microfibers revealed that U87 GBM cells significantly migrated for longer times $(27.06 \pm 1.65 \mathrm{~min})$ compared to HTB132 cells $(21.08 \pm 1.09 \mathrm{~min})$ (Fig. 8I). Addressing the total time of reverse migration occurring in one single cellular movement, U87 GBM cells demonstrated significantly fewer reverse movements $(6.22 \pm 0.83 \mathrm{~min})$ compared to HTB132 cells (9.96 $\pm 1.53 \mathrm{~min}$ ) (Fig. 8J). The above findings support that U87 GBM cells significantly migrated more persistently than HTB132.

The motor clutch model describes the physical and molecular mechanics of how cells move on substrates [53]. This complex process involves actin polymerization at the leading cell edge driving forward cell movement, and coordinated with actin depolymerizing proximally and centrally with localized myosin motors, which pull and exert force on this F-actin network, resulting in retrograde actin flow [54]. Clutch adaptor proteins like cell receptor integrins and other clutch components, like talin and vinculin, interact with F-actin, transmit forces to adhesion sites and regulate the cellular grip on the extracellular matrix controlling cell movement $[54,55]$. Previously it was shown that the molecular processes describing the motor-clutch model implemented many different genes [56-58] ( $n=$ genes): for clutch $(n=35)$, motor $(n=40)$, acceleration $(n=50)$ and migration impairment $(n=58)$. In line with the motor clutch model, we analyzed the relevant proteins mediating the motor clutch mechanism, as well as 
proposed proteins responsible for acceleration and impairment of migration according to our RNA-seq data [56-58]. Although some motor clutch specific genes were found higher expressed in U87 GBM grown in 3D with Matrigel and laminin-coated scaffolds and 2D cultures (Fig. 5C), our expression analyses using RNA-seq of the above 183 genes showed no significant differences (Fig. 8K).

\section{Discussion}

One of the first comparative histological analysis by Scherer in 1938 showed that GBM growth and dissemination throughout the brain depends upon the architectural organization [13]. Histologically he discovered that GBM cells were mainly associated with white matter axon bundles or could even follow dendrites when invading the cortex. Importantly, secondary tumors to the brain were mainly detected in border zone regions, like the cerebral vascular supply and gray and white matter junctions, further supporting that metastatic tumor cells travel along the arterial tree [59]. Brain structures in terms of stiffness are still under investigation. For example, local viscoelastic properties of neurons and glial cells using scanning force microscopy (SFM) showed that the elastic storage modulus ( $\mathrm{E}^{\prime}$ ) of astrocytes (glial cells) was between $\sim 300 \mathrm{~Pa}(30 \mathrm{~Hz})$ to $\sim 520 \mathrm{~Pa}(200 \mathrm{~Hz}$ ), whereas neurons ranged from $\sim 650 \mathrm{~Pa}$ (30 $\mathrm{Hz})$ to $\sim 1,590 \mathrm{~Pa}(200 \mathrm{~Hz})[60]$. These values defined the cells of the central nervous system (CNS) as very soft compared to other cells, like fibroblasts ( $\mathrm{E}^{\prime} \sim 3 \mathrm{kPa}$ at $\left.200 \mathrm{~Hz}\right)$ [61]. Axon bundle tracts have widths ranging from $0.1-10 \mu \mathrm{m}$ [62] and stiffnesses recorded of $4.6 \pm 1.5 \mathrm{kPa}$ (Young's modulus) [63]. Interestingly, using waveguide elastography with healthy individuals in vivo, similar values for white matter tracts of 4-4.6 kPa (shear modulus) were found [64]. It is well known that breast cancer tumors are stiffer $\left(E^{\prime}=4.04 \pm 0.9 \mathrm{kPa}\right)$ than normal mammary tissue $\left(E^{\prime}=0.167 \pm 0.031 \mathrm{kPa}\right)[65]$ supporting tumor cell mediated ECM remodeling. On the other hand single primary breast cancer cells are very soft ( $~ 300 \mathrm{~Pa}$ ) similar to CNS cells described above [66]. It is conceivable that tumor cells with different mechanical conditioning target different metastatic sites as shown for breast cancer cells [67]. Since it is known that cells have a high affinity for stiffer substrates [15], it is plausible that tumor cells demonstrate a durotactic activity for stiffer white matter tracts.

In the present study we implemented scaffolds and aligned microfibers (each $10 \mu \mathrm{m}$ ) with measured compression stiffnesses of $\sim 15 \mathrm{kPa}$ including porous spaces [39] to simulate axon tracts and blood vessels within the brain. Our findings bring forth novel information demonstrating that both GBM and TNBC cells with homozygote PTEN loss of function and high RHOB gene expression had similar 3D migration properties in contrast to GBM and TNBC cells with PTEN wt and low RHOB expression. Furthermore using TCGA analyses, $70 \%$ of primary GBM and breast cancer tumors with PTEN splice mutations had homozygote PTEN loss of function with significantly high RHOB expression (Fig. 5A). These primary tumors are similar to the cell lines used in this study, supporting a clinical relevance with our experimental findings regarding 3D migration. Thus, we conclude that the status of a specific tumor cell genotype together with expression of RHO / ROCK signaling genes represent "molecular cues" for 3D structures regulating durotaxis, cell adhesion and migration. 
Only GBM and TNBC cells with PTEN loss of function and high RHOB gene expression resulted in an enhanced single cell durotactic response, promoting cells to migrate towards stiffer scaffolds or aligned microfibers. Furthermore, following adherence of these tumor cells significantly led to a faster migration speed on aligned microfibers than PTEN wt and low RHOB expressing cells. It is noteworthy that HTB26 cells with PTEN wt and low RHOB expression showed a specific mode of "collective cell adherence" with aligned microfibers, where single cells could load onto and migrate at a slower speed. In contrast no durotaxis or migration occurred with LN18 GBM PTEN wt. Interestingly, an essential role for Pten controlling collective cell migration was also found during mouse embryonic development [68]. It was demonstrated that TGFB induced migration via PTEN suppression, where TGFB1 switched breast cancer cells from a collective to a single cell migration mode via RHO / ROCK signaling [69]. It will be important to further study the collective nature of HTB26 binding to aligned microfibers.

Another study using solution electrospun nanofiber scaffolds with a higher stiffness of up to $166 \mathrm{kPa}$ demonstrated increased migration of glioma stem cells depending on multibranched $\mathrm{N}$-glycans metabolized by MGAT5 [70]. According to our RNA-seq data of U87 GBM cells grown in 3D and scaffolds, with a 10-fold lower stiffness to the above study, the MGAT5 gene as well as other MGAT genes were not overexpressed implying that multibranched $\mathrm{N}$-glycans were probably not responsible for migration. In contrast to glioma stem cells, U87 GBM cells showed no overexpression of stem cell markers, like SOX2, ZEB1, ATXN1, ALCAM, CD9, ITGA7, CD44 and CHI3L1.

Although much is known regarding PTEN regulating chemotaxis and migration [71], we prove a pivotal role for PTEN controlling durotaxis and migration speed using aligned microfibers. Manipulating PTEN with a PTENi or rescuing PTEN function completely recovered durotaxis and migration of LN18 PTEN wt cells or blunted cell speed of U87 GBM, respectively. 2D studies of Tamura et al. showed that NIH3T3 mouse embryonic fibroblasts and U87 GBM cells overexpressing PTEN lowered 2D migration via reduced integrin mediated cell spreading and focal adhesions [72]. The latter study also showed that PTEN inhibition enhanced cell migration using 2D scratch assays. Furthermore, our findings reveal an essential role of RHO or downstream ROCK kinase activity for durotaxis and migration. Corroborating our U87 GBM RNA-seq, where RHOB was the highest significantly expressed gene (46.15-fold), which was confirmed with qPCR for U87 and HTB132, inhibition of RHO halted both durotaxis and migration without cell death. In addition, inhibiting ROCK also significantly blunted migration speed. Another ROCK inhibitor Fasudil ${ }^{\circledR}$ reduced invasion of GBM cells xenografted into mice [73]. In contrast, using HTB26 cells in confined environments like $3 \mu \mathrm{m}$ channels or GBM cells on 2D surfaces showed no inhibition of migration and speed with ROCK inhibitor Y-27632 supporting no Myosin II involvement using these conditions [74, 75]. Taken together, our above findings along with the literature support an essential regulatory role for the RHOB / ROCK / PTEN signaling pathway controlling durotaxis and migration.

Concerning cell morphology, Gong et al. showed that RHOB overexpression along with RHOB shuttling from endosomes to the plasma membrane led to cell blebbing and increased amoeboid migration in 3D collagen [33]. In contrast, a morphological switch from an amoeboid to a more elongated lamellipodial shaped cell involved RHOA signaling [52]. We also detected a significant switch of PTEN rescued U87 
GBM from an amoeboid shape to an elongated flat phenotype with a lower migration speed. Furthermore, we discovered that U87 GBM and HTB132 cells with homozygote PTEN loss of function and increased $R H O B$ expression had distinct amoeboid cell shapes as they migrate through a 3D matrix towards scaffolds (Figure S1A) and also on aligned microfibers (Fig. 7B,C, 8A-G). Further support for an amoeboid shape showed significant increased cell heights for U87 GBM and HTB132 cells, compared to more elongated cells with PTEN wt and RHOB low expression. Studies using normal mammary epithelial cells spread more on a stiff matrix $(5,000 \mathrm{~Pa})$ compared to a soft matrix of $140 \mathrm{~Pa}$, which was shown to be dependent on RHO, ROCK or Myosin [76].

Our confocal imaging of amoeboid shaped cells also showed enhancement of F-actin along the cell rim or cortex, which extended into different sized protrusions at the leading edge of the cell. The nucleus was mainly positioned in the cell rear. An enriched F-actin cortex has previously been noted in spherical-like shaped cells presenting a single barrier along the cell circumference [77]. It is notable that RHOB recruits DIAPH1 to endosomes forming an F-actin coat [78]. It would be interesting to analyze if RHOB and DIAPH1 also play a role in F-actin membrane coating, due to the fact that RHOB shuttles between endosomes and plasma membrane [33]. Increased actin-myosin contraction also associates with highly motile round cancer cells along with decreased adhesion, thus supporting a basis for our observations of fast amoeboid cell movements on aligned microfibers with less cell surface area [79].

It is known that different cellular protrusions can be found associated with amoeboid shaped cells contributing to cell movement [80]. It was shown that single cellular focal adhesions autonomously sense the environment for stiffness and rigidity [15]. The latter study proved that the sensing of focal adhesions via the Paxillin (PXN)/ Vinculin (VCL)/ FAK (PTK2) pathway was essential for durotaxis, but not chemotaxis. Our RNA-seq of U87 GBM cells in 3D with scaffolds versus 2D showed an induction of the adhesion adaptor gene PXN (2.46-fold), but not of VCL and PTK2. Additionally, it was shown that soft matrices suppressed actin-myosin assembly and orientation. Specifically, myosin heavy chain IIA (MYH9) initiated formation of actin-myosin filaments and myosin heavy chain IIB (MYH10) bound and stabilized these filaments. In contrast to soft matrices, MYH9 did not polarize, but MYH10 was induced during durotaxis with stiff substrates [81], which we found 7.5-fold upregulated with our RNA-seq of U87 GBM in $3 \mathrm{D}$ with scaffolds. Lastly, regarding the nucleus position of migrating cells, it was shown that the direction of cell migration appeared to be driven by positioning the nucleus towards the cell rear in multiple cell types via microtubules with a possible extension of the leading edge [82]. Rearward orientation of the nucleus was also regulated by activation of RHO signaling pathways [83].

Our SEM findings showing alignment of single amoeboid cells on individual $10 \mu \mathrm{m}$ fibers within a single $100 \mu \mathrm{m}$ aligned microfiber tract, support a topographical cue for migration (Fig. 3B, 7B). On the other hand, elongated cells appeared to topographically sense and stretch over several PCL fibers. Although, the majority of topotaxis studies address cells sensing specific 3D nanostructures [18], our laminincoated aligned microfibers represent smooth $10 \mu \mathrm{m}$ round tube-like structures. Lastly, for both amoeboid U87 GBM and HTB132 cells with high RHOB expression and PTEN loss of function, we recorded different plasticity modes, which we coined "skating snails". For example, U87 GBM cell surface lengths along the 
aligned microfibers corresponded with different speeds. Fast migrating U87 GBM cells showed a surface length of $10 \mu \mathrm{m}$, whereas slow and halted U87 GBM cells were $20 \mu \mathrm{m}$ or $30 \mu \mathrm{m}$ in length, respectively (Fig. 8H). In line with our findings, Maiuri et al. showed that cell speed and persistence are exponentially coupled via actin flow [84]. Although, U87 GBM and HTB132 showed similar plasticity modes, U87 GBM cells were more persistent "skating" for longer distances than HTB132 cells. Thus we support the idea that these cellular plasticity modes reflect intrinsic genetic differences between GBM and TNBC cells.

Other publications have also implemented biomaterials or ECM fiber structures to mimic brain structures, like axons, to investigate tumor cell migration in the brain [47, 85-87]. All these publications tested fiber sizes, fiber widths, single, stacked or parallel fibers to resemble axon and blood vessel structures. One common finding among all studies was the striking different plasticity modes of cells migrating on various fiber constructs. Doyle et al. demonstrated that cells migrating on $1.5 \mu \mathrm{m}$ single fibrillar lines (also called 1D) composed of fibronectin was identical to cells migrating in a 3D fibrillar matrix, but were significantly slower on 2D fibronectin coated substrates [85]. The authors concluded that both 1D and 3D cellular migration were comparable based upon different properties of migrating cells. For example, cells had a uniaxial cell morphology with a single lamellipodia and a coordinated protrusion-retraction cycle, which resulted in a unidirectional rapid migration independent of ECM density, but dependent on myosin II contractility.

\section{Conclusions}

Our findings validate a significant role of specific PTEN genotypes along with RHOB signaling controlling cell morphology, durotaxis, adhesion, cellular plasticity and migration speed of tumor cells. For both GBM and TNBC types with homozygote PTEN loss of function and high RHOB expression we propose that the cellular plasticity modes of amoeboid cells promote enhanced durotaxis, increased migratory speeds and longer traveling distances, which could lead to faster dissemination throughout the brain. The fact that PTEN regulates the cell polarity of the PIP3 / PIP2 gradient, supports the idea that an aberrant gradient due to PTEN loss of function could be responsible for the above deregulations. Presently clinical treatments have not yet achieved the goal to extend the survival of patients with GBM or breast cancer patients with metastasizing tumors in the brain. Our study demonstrates the importance of PTEN and RHOB not only for primary tumors, but also for metastasizing tumors, where RHOB inhibitors could play an essential role for improved therapy.

\section{Abbreviations}

GBM: Glioblastoma multiforme; E': elastic storage modulus; ECM: extracellular matrix; MEW: melt electrowriting; PI3K: phosphoinositide 3-kinase; PCL: poly-( $\varepsilon$-caprolactone); PIP2: phosphatidylinositol4,5-bisphosphate; PIP3: phosphatidylinositol-3,4,5-triphosphate; PTEN: Phosphatase and Tensin Homolog; PTENi: PTEN inhibitor; RHOB: ras homolog family member B; RHOi = RHO inhibitor; ROCK: rho associated coiled-coil containing protein kinase; ROCKi: ROCK inhibitor; RNA-seq: RNA-sequencing; wt: wildtype; SFM: scanning force microscopy; TNBC: triple-negative breast cancer. 


\section{Declarations}

\section{Ethics approval and consent to participate}

Not applicable

\section{Consent for publication}

Not applicable

\section{Availability of data and materials}

The datasets used and/or analyzed during the current study are available from the corresponding author on reasonable request.

\section{Competing interests}

The authors declare that they have no competing interests.

\section{Funding}

This research project was funded by the Deutsche Forschungsgemeinschaft (DFG, German Research Foundation) - Project number 326998133 - TRR 225 (subproject B01).

\section{Authors' contributions}

RS and PS conceived, designed and coordinated the study; AW and HS designed and performed the experiments; EB and PD designed and produced the biomaterials; DJ performed 3D computer reconstructions from confocal images; AW and RS prepared the figures; ME performed the TCGA analysis; RS and PS wrote and AW helped to finalize the manuscript; AW, HS, EB, PD, DJ, ME, MB, RS and PS edited the manuscript. All authors read and approved the final manuscript.

\section{Acknowledgements}

Technical assistance of Lena Fischer and Adrian Weich was greatly appreciated.

\section{References}

1. Hanahan D, Weinberg RA. Hallmarks of cancer: the next generation. Cell. 2011;144:646-74.

2. Ridley AJ. Rho GTPase signalling in cell migration. Curr Opin Cell Biol. 2015;36:103-12.

3. Friedl P, Sahai E, Weiss S, Yamada KM. New dimensions in cell migration. Nat Rev Mol Cell Biol. 2012;13:743-7.

4. Papusheva E, Heisenberg CP. Spatial organization of adhesion: force-dependent regulation and function in tissue morphogenesis. EMBO J. 2010;29:2753-68. 
5. Frantz C, Stewart KM, Weaver VM. The extracellular matrix at a glance. J Cell Sci. 2010;123:4195200.

6. Pedron S, Becka E, Harley BA. Regulation of glioma cell phenotype in 3D matrices by hyaluronic acid. Biomaterials. 2013;34:7408-17.

7. Ulrich TA, de Juan Pardo EM, Kumar S. The mechanical rigidity of the extracellular matrix regulates the structure, motility, and proliferation of glioma cells. Cancer Res. 2009;69:4167-74.

8. Mair DB, Ames HM, Li R. Mechanisms of invasion and motility of high-grade gliomas in the brain. Mol Biol Cell. 2018;29:2509-15.

9. Vega FM, Fruhwirth G, Ng T, Ridley AJ. RhoA and RhoC have distinct roles in migration and invasion by acting through different targets. J Cell Biol. 2011;193:655-65.

10. Rossi J, Giaccherini L, Cavallieri F, Napoli M, Moratti C, Froio E, et al. Extracranial metastases in secondary glioblastoma multiforme: a case report. BMC Neurol. 2020;20:382.

11. Hara A, Kanayama T, Noguchi K, Niwa A, Miyai M, Kawaguchi M, et al. Treatment Strategies Based on Histological Targets against Invasive and Resistant Glioblastoma. J Oncol. 2019;2019:2964783.

12. Thakkar JP, Dolecek TA, Horbinski C, Ostrom QT, Lightner DD, Barnholtz-Sloan JS, et al. Epidemiologic and molecular prognostic review of glioblastoma. Cancer Epidemiol Biomarkers Prev. 2014;23:1985-96.

13. Scherer HJ. STRUCTURAL DEVELOPMENT IN GLIOMAS. The American Journal of Cancer. 1938;34:333-51.

14. Farin A, Suzuki SO, Weiker M, Goldman JE, Bruce JN, Canoll P. Transplanted glioma cells migrate and proliferate on host brain vasculature: a dynamic analysis. Glia. 2006;53:799-808.

15. Plotnikov SV, Pasapera AM, Sabass B, Waterman CM. Force fluctuations within focal adhesions mediate ECM-rigidity sensing to guide directed cell migration. Cell. 2012;151:1513-27.

16. SenGupta S, Parent CA, Bear JE. The principles of directed cell migration. Nat Rev Mol Cell Biol. 2021;22:529-47.

17. Caballero D, Comelles J, Piel M, Voituriez R, Riveline D. Ratchetaxis: Long-Range Directed Cell Migration by Local Cues. Trends Cell Biol. 2015;25:815-27.

18. Park J, Kim DH, Kim HN, Wang CJ, Kwak MK, Hur E, et al. Directed migration of cancer cells guided by the graded texture of the underlying matrix. Nat Mater. 2016;15:792-801.

19. Lv Y, Ma X, Du Y, Feng J. Understanding Patterns of Brain Metastasis in Triple-Negative Breast Cancer and Exploring Potential Therapeutic Targets. Onco Targets Ther. 2021;14:589-607.

20. Brosnan EM, Anders CK. Understanding patterns of brain metastasis in breast cancer and designing rational therapeutic strategies. Ann Transl Med. 2018;6:163.

21. Leslie NR, Yang X, Downes CP, Weijer CJ. Ptdlns(3,4,5)P(3)-dependent and -independent roles for PTEN in the control of cell migration. Curr Biol. 2007;17:115-25.

22. Alvarez-Garcia V, Tawil Y, Wise HM, Leslie NR. Mechanisms of PTEN loss in cancer: It's all about diversity. Semin Cancer Biol. 2019;59:66-79. 
23. Ohgaki H, Dessen P, Jourde B, Horstmann S, Nishikawa T, Di Patre PL, et al. Genetic pathways to glioblastoma: a population-based study. Cancer Res. 2004;64:6892-9.

24. Furukawa K, Kumon $Y$, Harada $H$, Kohno S, Nagato S, Teraoka $M$, et al. PTEN gene transfer suppresses the invasive potential of human malignant gliomas by regulating cell invasion-related molecules. Int J Oncol. 2006;29:73-81.

25. Witzel I, Oliveira-Ferrer L, Pantel K, Muller V, Wikman H. Breast cancer brain metastases: biology and new clinical perspectives. Breast Cancer Res. 2016;18:8.

26. Csolle MP, Ooms LM, Papa A, Mitchell CA. PTEN and Other Ptdlns(3,4,5)P3 Lipid Phosphatases in Breast Cancer. Int J Mol Sci. 2020;21.

27. Milella M, Falcone I, Conciatori F, Cesta Incani U, Del Curatolo A, Inzerilli N, et al. PTEN: Multiple Functions in Human Malignant Tumors. Front Oncol. 2015;5:24.

28. Hohensee I, Chuang HN, Grottke A, Werner S, Schulte A, Horn S, et al. PTEN mediates the cross talk between breast and glial cells in brain metastases leading to rapid disease progression. Oncotarget. 2017;8:6155-68.

29. Wikman H, Lamszus K, Detels N, Uslar L, Wrage M, Benner C, et al. Relevance of PTEN loss in brain metastasis formation in breast cancer patients. Breast Cancer Res. 2012;14:R49.

30. Adamo B, Deal AM, Burrows E, Geradts J, Hamilton E, Blackwell KL, et al. Phosphatidylinositol 3kinase pathway activation in breast cancer brain metastases. Breast Cancer Res. 2011;13:R125.

31. Lawson CD, Ridley AJ. Rho GTPase signaling complexes in cell migration and invasion. J Cell Biol. 2018;217:447-57.

32. Li Z, Dong X, Wang Z, Liu W, Deng N, Ding Y, et al. Regulation of PTEN by Rho small GTPases. Nat Cell Biol. 2005;7:399-404.

33. Gong X, Didan Y, Lock JG, Stromblad S. KIF13A-regulated RhoB plasma membrane localization governs membrane blebbing and blebby amoeboid cell migration. EMBO J. 2018;37.

34. Loessner D, Rockstroh A, Shokoohmand A, Holzapfel BM, Wagner F, Baldwin J, et al. A 3D tumor microenvironment regulates cell proliferation, peritoneal growth and expression patterns. Biomaterials. 2019;190-191:63-75.

35. Hutmacher DW, Loessner D, Rizzi S, Kaplan DL, Mooney DJ, Clements JA. Can tissue engineering concepts advance tumor biology research? Trends in Biotechnology. 2010;28:125-33.

36. Kim J, Bakirci E, O'Neill KL, Hrynevich A, Dalton PD. Fiber Bridging during Melt Electrowriting of Poly(epsilon-Caprolactone) and the Influence of Fiber Diameter and Wall Height. Macromol Mater Eng. 2021;306.

37. Pham QP, Sharma U, Mikos AG. Electrospinning of polymeric nanofibers for tissue engineering applications: a review. Tissue Eng. 2006;12:1197-211.

38. Robinson TM, Hutmacher DW, Dalton PD. The Next Frontier in Melt Electrospinning: Taming the Jet. Advanced Functional Materials. 2019;29:1904664. 
39. Visser J, Melchels FP, Jeon JE, van Bussel EM, Kimpton LS, Byrne HM, et al. Reinforcement of hydrogels using three-dimensionally printed microfibres. Nat Commun. 2015;6:6933.

40. Janzen D, Bakirci E, Wieland A, Martin C, Dalton PD, Villmann C. Cortical Neurons form a Functional Neuronal Network in a 3D Printed Reinforced Matrix. Adv Healthc Mater. 2020;9:e1901630.

41. Weigand A, Boos AM, Tasbihi K, Beier JP, Dalton PD, Schrauder M, et al. Selective isolation and characterization of primary cells from normal breast and tumors reveal plasticity of adipose derived stem cells. Breast Cancer Res. 2016;18:32.

42. Furnari FB, Lin H, Huang HS, Cavenee WK. Growth suppression of glioma cells by PTEN requires a functional phosphatase catalytic domain. Proc Natl Acad Sci U S A. 1997;94:12479-84.

43. Hollestelle A, Elstrodt F, Nagel JH, Kallemeijn WW, Schutte M. Phosphatidylinositol-3-OH kinase or RAS pathway mutations in human breast cancer cell lines. Mol Cancer Res. 2007;5:195-201.

44. Grosshaus C, Bakirci E, Berthel M, Hrynevich A, Kade JC, Hochleitner G, et al. Melt Electrospinning of Nanofibers from Medical-Grade Poly(epsilon-Caprolactone) with a Modified Nozzle. Small. 2020;16:e2003471.

45. Cerami E, Gao J, Dogrusoz U, Gross BE, Sumer So, Aksoy BA, et al. The cBio cancer genomics portal: an open platform for exploring multidimensional cancer genomics data. Cancer Discov. 2012;2:4014.

46. Bakirci E, Schaefer N, Dahri O, Hrynevich A, Strissel P, Strick R, et al. Melt Electrowritten In Vitro Radial Device to Study Cell Growth and Migration. Adv Biosyst. 2020;4:e2000077.

47. Jain A, Betancur M, Patel GD, Valmikinathan CM, Mukhatyar VJ, Vakharia A, et al. Guiding intracortical brain tumour cells to an extracortical cytotoxic hydrogel using aligned polymeric nanofibres. Nat Mater. 2014;13:308-16.

48. Lo CM, Wang HB, Dembo M, Wang YL. Cell movement is guided by the rigidity of the substrate. Biophys J. 2000;79:144-52.

49. DuChez BJ, Doyle AD, Dimitriadis EK, Yamada KM. Durotaxis by Human Cancer Cells. Biophys J. 2019;116:670-83.

50. Yousif LF, Di Russo J, Sorokin L. Laminin isoforms in endothelial and perivascular basement membranes. Cell Adh Migr. 2013;7:101-10.

51. Lau LW, Cua R, Keough MB, Haylock-Jacobs S, Yong VW. Pathophysiology of the brain extracellular matrix: a new target for remyelination. Nat Rev Neurosci. 2013;14:722-9.

52. Yamada KM, Sixt M. Mechanisms of 3D cell migration. Nat Rev Mol Cell Biol. 2019;20:738-52.

53. Mitchison T, Kirschner M. Cytoskeletal dynamics and nerve growth. Neuron. 1988;1:761-72.

54. Giannone G, Dubin-Thaler BJ, Rossier O, Cai Y, Chaga O, Jiang G, et al. Lamellipodial actin mechanically links myosin activity with adhesion-site formation. Cell. 2007;128:561-75.

55. Riveline D, Zamir E, Balaban NQ, Schwarz US, Ishizaki T, Narumiya S, et al. Focal contacts as mechanosensors: externally applied local mechanical force induces growth of focal contacts by an mDia1-dependent and ROCK-independent mechanism. J Cell Biol. 2001;153:1175-86. 
56. Bangasser BL, Odde DJ. Master equation-based analysis of a motor-clutch model for cell traction force. Cell Mol Bioeng. 2013;6:449-59.

57. Prahl LS, Bangasser PF, Stopfer LE, Hemmat M, White FM, Rosenfeld SS, et al. Microtubule-Based Control of Motor-Clutch System Mechanics in Glioma Cell Migration. Cell Rep. 2018;25:2591-604 e8.

58. Simpson KJ, Selfors LM, Bui J, Reynolds A, Leake D, Khvorova A, et al. Identification of genes that regulate epithelial cell migration using an siRNA screening approach. Nat Cell Biol. 2008;10:1027-38.

59. Hwang TL, Close TP, Grego JM, Brannon WL, Gonzales F. Predilection of brain metastasis in gray and white matter junction and vascular border zones. Cancer. 1996;77:1551-5.

60. Lu YB, Franze K, Seifert G, Steinhauser C, Kirchhoff F, Wolburg H, et al. Viscoelastic properties of individual glial cells and neurons in the CNS. Proc Natl Acad Sci U S A. 2006;103:17759-64.

61. Mahaffy RE, Shih CK, MacKintosh FC, Kas J. Scanning probe-based frequency-dependent microrheology of polymer gels and biological cells. Phys Rev Lett. 2000;85:880-3.

62. Costa AR, Pinto-Costa R, Sousa SC, Sousa MM. The Regulation of Axon Diameter: From Axonal Circumferential Contractility to Activity-Dependent Axon Swelling. Front Mol Neurosci. 2018;11:319.

63. Zhang Y, Abiraman K, Li H, Pierce DM, Tzingounis AV, Lykotrafitis G. Modeling of the axon membrane skeleton structure and implications for its mechanical properties. PLoS Comput Biol. 2017;13:e1005407.

64. Romano A, Scheel M, Hirsch S, Braun J, Sack I. In vivo waveguide elastography of white matter tracts in the human brain. Magn Reson Med. 2012;68:1410-22.

65. Paszek MJ, Zahir N, Johnson KR, Lakins JN, Rozenberg GI, Gefen A, et al. Tensional homeostasis and the malignant phenotype. Cancer Cell. 2005;8:241-54.

66. Lautscham LA, Kammerer C, Lange JR, Kolb T, Mark C, Schilling A, et al. Migration in Confined 3D Environments Is Determined by a Combination of Adhesiveness, Nuclear Volume, Contractility, and Cell Stiffness. Biophys J. 2015;109:900-13.

67. Watson AW, Grant AD, Parker SS, Hill S, Whalen MB, Chakrabarti J, et al. Breast tumor stiffness instructs bone metastasis via maintenance of mechanical conditioning. Cell Rep. 2021;35:109293.

68. Bloomekatz J, Grego-Bessa J, Migeotte I, Anderson KV. Pten regulates collective cell migration during specification of the anterior-posterior axis of the mouse embryo. Dev Biol. 2012;364:192-201.

69. Giampieri S, Manning C, Hooper S, Jones L, Hill CS, Sahai E. Localized and reversible TGFbeta signalling switches breast cancer cells from cohesive to single cell motility. Nat Cell Biol. 2009;11:1287-96.

70. Marhuenda E, Fabre C, Zhang C, Martin-Fernandez M, Iskratsch T, Saleh A, et al. Glioma stem cells invasive phenotype at optimal stiffness is driven by MGAT5 dependent mechanosensing. J Exp Clin Cancer Res. 2021;40:139.

71. Funamoto S, Meili R, Lee S, Parry L, Firtel RA. Spatial and temporal regulation of 3-phosphoinositides by PI 3-kinase and PTEN mediates chemotaxis. Cell. 2002;109:611-23. 
72. Tamura M, Gu J, Matsumoto K, Aota S, Parsons R, Yamada KM. Inhibition of cell migration, spreading, and focal adhesions by tumor suppressor PTEN. Science. 1998;280:1614-7.

73. Deng L, Li G, Li R, Liu Q, He Q, Zhang J. Rho-kinase inhibitor, fasudil, suppresses glioblastoma cell line progression in vitro and in vivo. Cancer Biol Ther. 2010;9:875-84.

74. Balzer EM, Tong Z, Paul CD, Hung WC, Stroka KM, Boggs AE, et al. Physical confinement alters tumor cell adhesion and migration phenotypes. FASEB J. 2012;26:4045-56.

75. Smith CL, Kilic O, Schiapparelli P, Guerrero-Cazares H, Kim DH, Sedora-Roman NI, et al. Migration Phenotype of Brain-Cancer Cells Predicts Patient Outcomes. Cell Rep. 2016;15:2616-24.

76. Butcher DT, Alliston T, Weaver VM. A tense situation: forcing tumour progression. Nat Rev Cancer. 2009;9:108-22.

77. Charras GT, Hu CK, Coughlin M, Mitchison TJ. Reassembly of contractile actin cortex in cell blebs. J Cell Biol. 2006;175:477-90.

78. Fernandez-Borja M, Janssen L, Verwoerd D, Hordijk P, Neefjes J. RhoB regulates endosome transport by promoting actin assembly on endosomal membranes through Dia1. J Cell Sci. 2005;118:2661-70.

79. Deakin NO, Turner CE. Distinct roles for paxillin and Hic-5 in regulating breast cancer cell morphology, invasion, and metastasis. Mol Biol Cell. 2011;22:327-41.

80. Petrie RJ, Harlin HM, Korsak LI, Yamada KM. Activating the nuclear piston mechanism of 3D migration in tumor cells. J Cell Biol. 2017;216:93-100.

81. Raab M, Swift J, Dingal PC, Shah P, Shin JW, Discher DE. Crawling from soft to stiff matrix polarizes the cytoskeleton and phosphoregulates myosin-II heavy chain. J Cell Biol. 2012;199:669-83.

82. Gundersen GG, Worman HJ. Nuclear positioning. Cell. 2013;152:1376-89.

83. Maninova M, Klimova Z, Parsons JT, Weber MJ, Iwanicki MP, Vomastek T. The reorientation of cell nucleus promotes the establishment of front-rear polarity in migrating fibroblasts. J Mol Biol. 2013;425:2039-55.

84. Maiuri P, Rupprecht JF, Wieser S, Ruprecht V, Benichou O, Carpi N, et al. Actin flows mediate a universal coupling between cell speed and cell persistence. Cell. 2015;161:374-86.

85. Doyle AD, Wang FW, Matsumoto K, Yamada KM. One-dimensional topography underlies threedimensional fibrillar cell migration. J Cell Biol. 2009;184:481-90.

86. Sharma P, Sheets K, Elankumaran S, Nain AS. The mechanistic influence of aligned nanofibers on cell shape, migration and blebbing dynamics of glioma cells. Integr Biol (Camb). 2013;5:1036-44.

87. Estabridis HM, Jana A, Nain A, Odde DJ. Cell Migration in 1D and 2D Nanofiber Microenvironments. Ann Biomed Eng. 2018;46:392-403.

88. Fukunaga T, Fujita Y, Kishima H, Yamashita T. Methylation dependent down-regulation of G0S2 leads to suppression of invasion and improved prognosis of IDH1-mutant glioma. PLoS One. 2018;13:e0206552.

89. Podgorska M, Pietraszek-Gremplewicz K, Nowak D. Apelin Effects Migration and Invasion Abilities of Colon Cancer Cells. Cells. 2018;7. 
90. Tochio T, Tanaka H, Nakata S, Hosoya H. Fructose-1,6-bisphosphate aldolase A is involved in HaCaT cell migration by inducing lamellipodia formation. J Dermatol Sci. 2010;58:123-9.

91. Messina A, Ferraris N, Wray S, Cagnoni G, Donohue DE, Casoni F, et al. Dysregulation of Semaphorin7A/beta1-integrin signaling leads to defective $\mathrm{GnRH}-1$ cell migration, abnormal gonadal development and altered fertility. Hum Mol Genet. 2011;20:4759-74.

92. Duan S, Huang W, Liu X, Liu X, Chen N, Xu Q, et al. IMPDH2 promotes colorectal cancer progression through activation of the PI3K/AKT/mTOR and PI3K/AKT/FOXO1 signaling pathways. J Exp Clin Cancer Res. 2018;37:304.

\section{Figures}

A

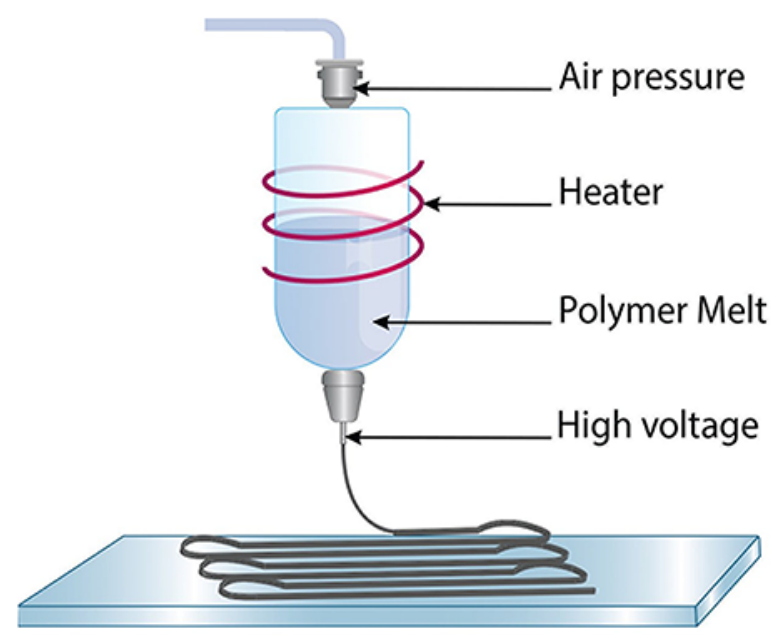

C

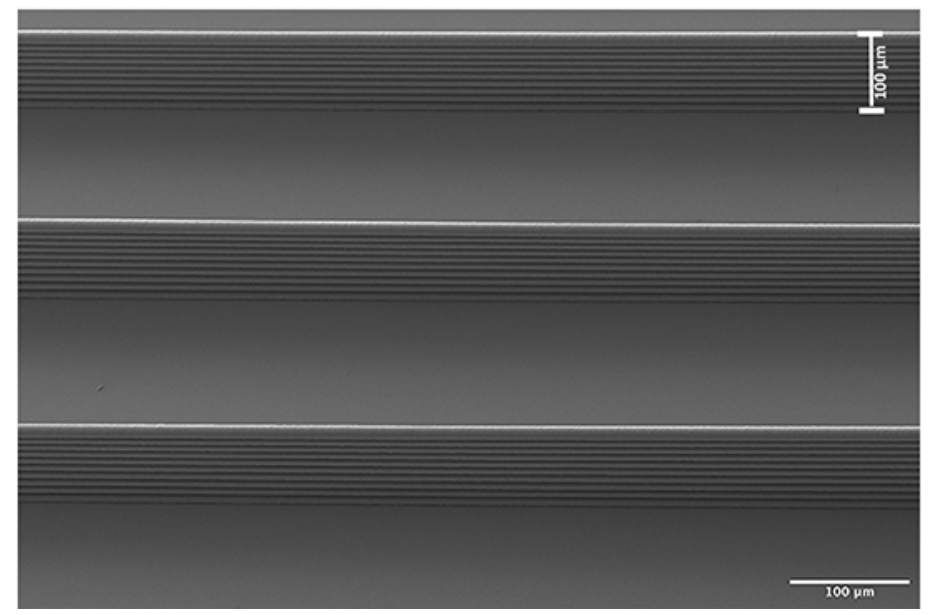

B

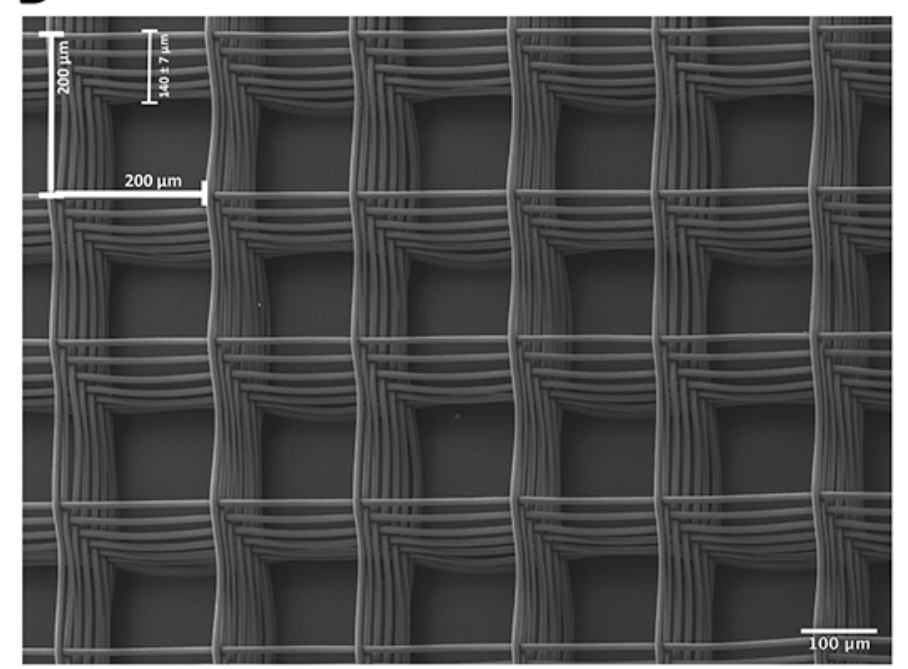

D

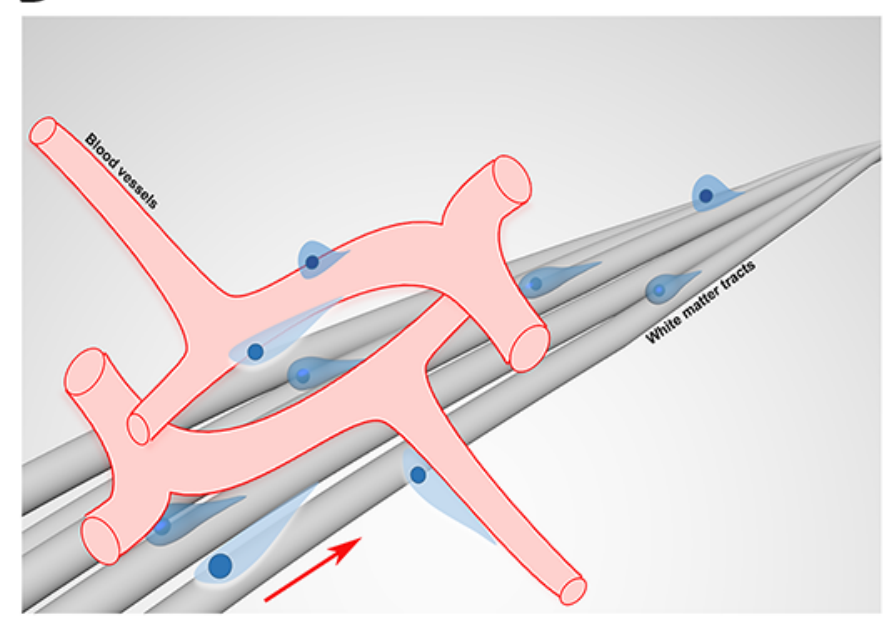

\section{Figure 1}

Fabrication and design of 3D scaffolds and aligned microfibers have similar properties. A) Schematic representation of MEW. Medical grade PCL melt was extruded through an electrified nozzle into a jet that solidified upon reaching the collector. Repeated deposition enabled the fabrication of a $9 \mathrm{~mm}$ diameter 
scaffold or an $8 \mathrm{~mm}$ long array of aligned microfibers. B) Scanning electron microscopy (SEM) image of a scaffold consisting of 10 stacked aligned fibers along the length and 20 stacked overlapping aligned fibers at each corner, where each fiber has a diameter of $10 \mu \mathrm{m}$. The inter-fiber bundle distance is $200 \mu \mathrm{m}$ (indicated as a white bar) with an overall box form scaffold structure with the total height of the fiber walls $\sim 140 \pm 7 \mu \mathrm{m}$ as indicated (in white). C) SEM image of an aligned microfiber. Each fiber bundle tract consists of 10 stacked aligned fibers where each PCL fiber is $10 \mu \mathrm{m}$ in diameter with the total height of the fiber walls $106 \pm 15 \mu \mathrm{m}$ (indicated as a white bar). The inter-fiber bundle distance is $200 \mu \mathrm{m}$. D) Schematic model representation of white matter tracts (grey) and blood vessels (pink) as topographical migratory cues for GBM tumor cells (blue) disseminating throughout the brain. The red arrow shows the direction of tumor cells with a protrusion at the leading edge and the nucleus at the rear of the cell. 
A

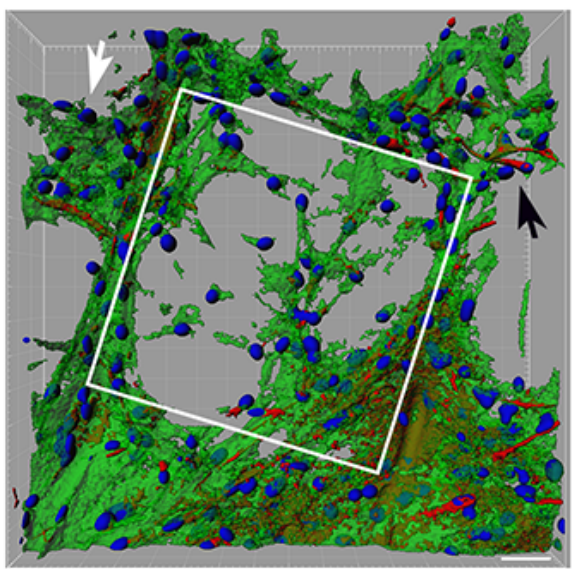

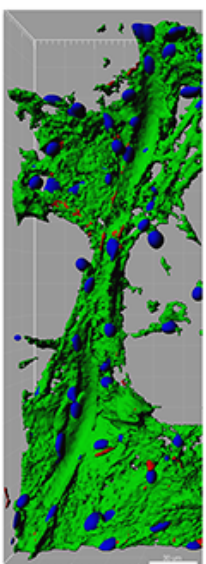

$5 \mathrm{~h}$

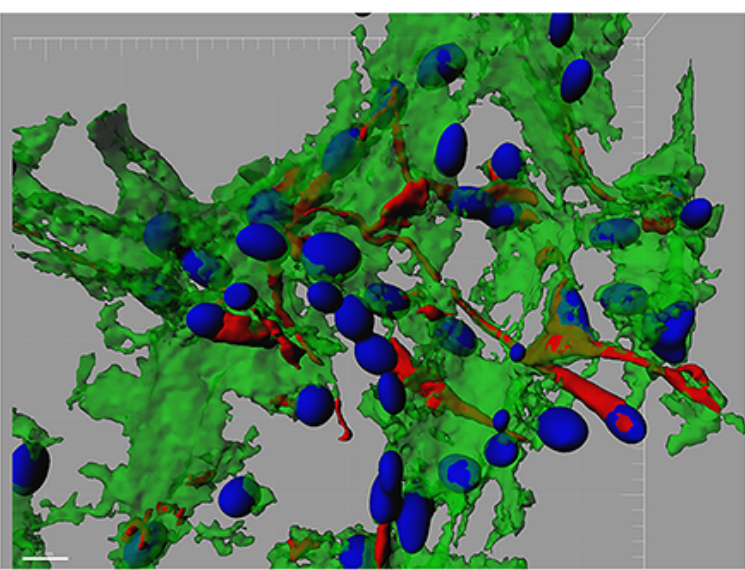

$10 \mathrm{~h}$

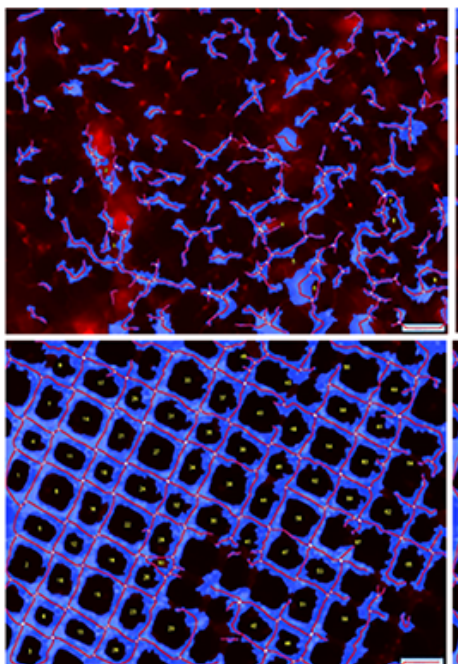

$18 \mathrm{~h}$
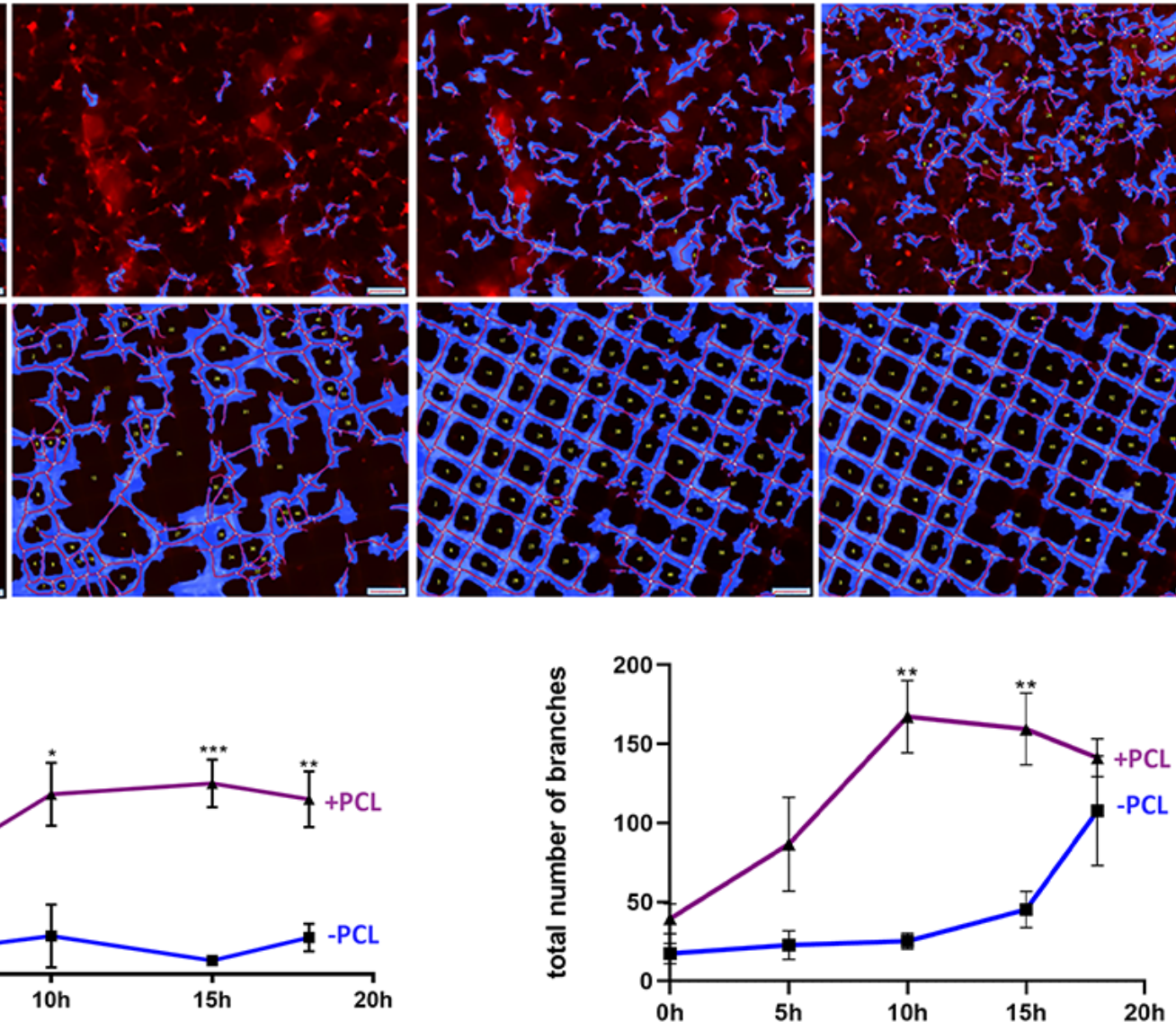

Figure 2

U87 GBM durotaxis and cell network formation with scaffolds in 3D cultures. A) Left image of a 3D reconstruction of an immunocytochemical staining of U87 GBM cells at day 6 with $4.5 \mathrm{mg} / \mathrm{ml}-1$ Matrigel and a single box pore $(200 \mu \mathrm{m}$ ) scaffold (white solid square) (white scale bar $=30 \mu \mathrm{m}$ ) (ß-tubulin, green; MAP2, red; Hoechst 33342, blue nuclei). The middle image shows the same region from the left image (white arrow) but without the scaffold to show the wrapping of U87 GBM cells around the aligned microfibers. Right image is a magnification of the left image (black arrow) to demonstrate U87 GBM cell interactions at day 6 (right scale bar $=10 \mu \mathrm{m}$ ) (ß-tubulin, green; MAP2, red; Hoechst 33342, blue nuclei). 
B) Cell network formation assay. U87-td-farnesyl expressing cells grown in 3D with $4.5 \mathrm{mg} \mathrm{ml}-1$ Matrigel alone (-PCL above) or with a scaffold (+PCL bottom) and imaged over time up to $18 \mathrm{~h}$. Images were analyzed for cell branches or loop-like structures (blue with red lines) using the WimTube computer program. White scale bar $=200 \mu \mathrm{m}$. C) Graphs show cell network formation assay (+PCL) scaffolds top red line ( $n=6$ experiments) and (-PCL) scaffolds bottom blue line ( $n=8$ experiments) quantified as the total number of cell loops (left graph, Y-axis) and branches (right graph, Y-axis) formed over $18 \mathrm{~h}$.

Significance was found comparing +PCL to -PCL; cell loops at $10 \mathrm{~h} * p=0.0113 ; 15 \mathrm{~h} * \star \star p=0.0007 ; 18 \mathrm{~h}$ $\star * p=0.0023$; cell branches at $10 h * * p=0.002 ; 15 h * * p=0.0079$ ). 

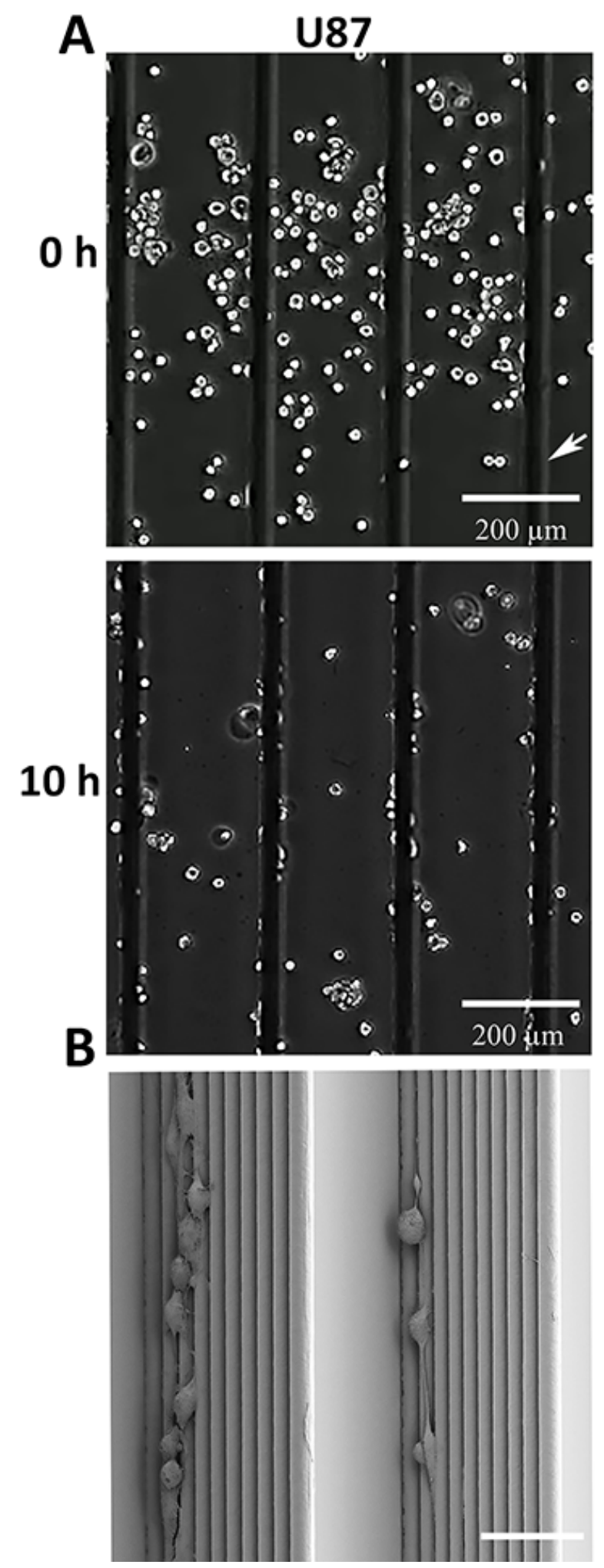

Figure 3

U87 GBM durotaxis and cell migration on aligned microfibers. A) Representative images of U87 GBM cells on laminin-coated aligned microfibers at $0 \mathrm{~h}$ and $10 \mathrm{~h}$; scale bar $=200 \mu \mathrm{m}$. B) Representative SEMimage of U87 GBM cells on stacked aligned microfibers (total 10 MEW printed fibers; scale bar $=20 \mu \mathrm{m}$ ). 
A
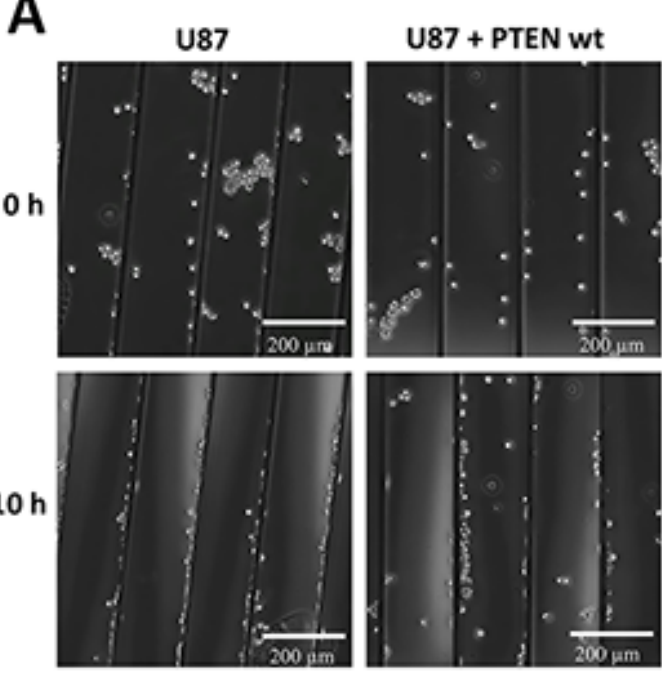

C

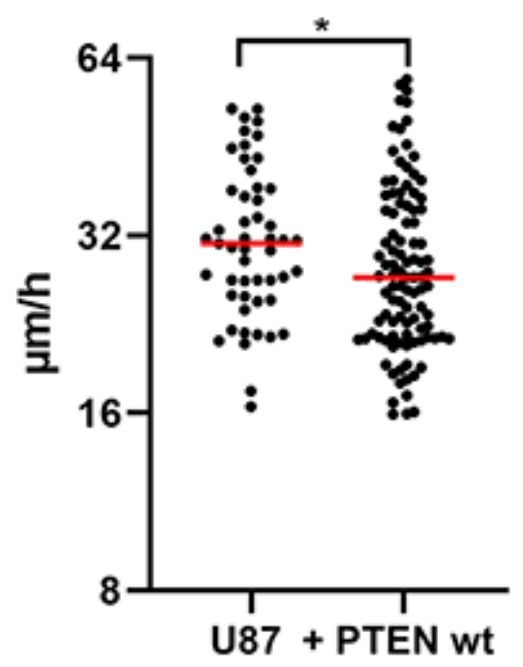

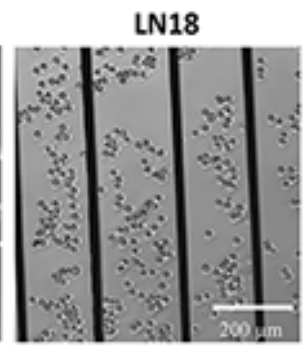
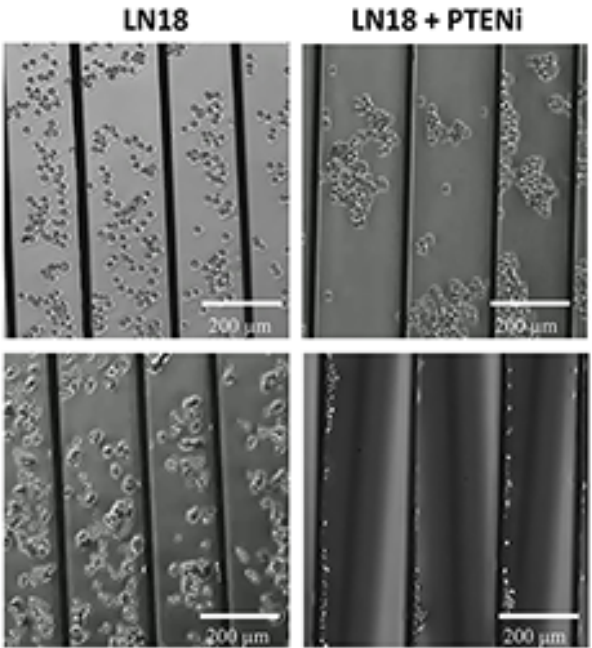

D

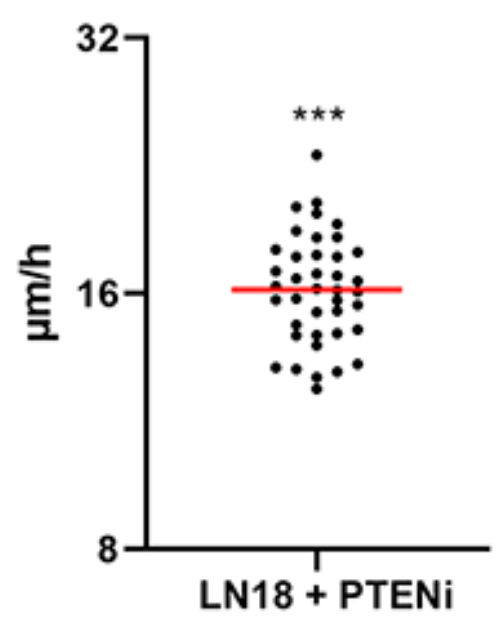

B
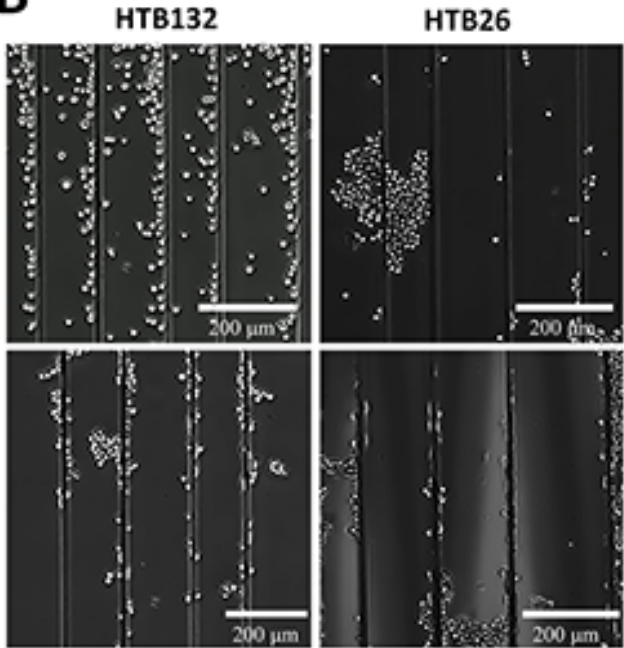

E

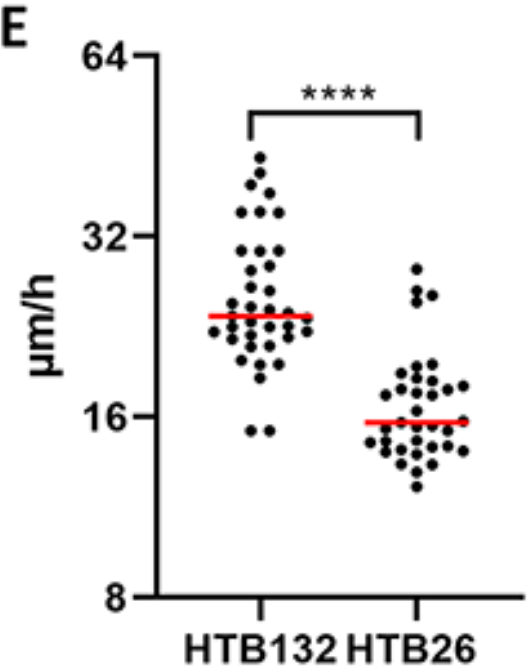

Figure 4

PTEN regulates GBM and TNBC cell durotaxis and migration. A) Representative images from left to right of U87 GBM PTEN loss of function cells (1st column, control) and U87 GBM cells after PTEN wt transfection and rescue (2nd column) on laminin-coated aligned microfibers at $0 \mathrm{~h}$ and $10 \mathrm{~h}$ (scale bar = $200 \mu \mathrm{m}$ ). LN18 GBM PTEN wt cells (3rd column, control), and LN18 GBM cells (4th column) following PTENi SF1670 treatment on laminin-coated aligned microfibers at $0 \mathrm{~h}$ and $10 \mathrm{~h}$. B) Representative images of HTB132 PTEN loss of function cells and HTB26 PTEN wt cells on laminin-coated aligned microfibers at $0 \mathrm{~h}$ and $10 \mathrm{~h}$ (scale bar $=200 \mu \mathrm{m}$ ). Note the specific collective adherence at $0 \mathrm{~h}$ of HTB26 PTEN wt cells $\mathrm{C})$ Aligned microfiber migration speed $(\mu \mathrm{m} / \mathrm{h})$ of U87 GBM cells PTEN loss of function (control) ( $\mathrm{n}=4$ experiments; $\mathrm{n}=51$ cells) and U87 GBM cells after PTEN wt transfection and rescue $(\mathrm{n}=3$ experiments; $n=101$ cells $) * p=0.0341$. D) Migration speed $(\mu \mathrm{m} / \mathrm{h})$ of LN18 GBM PTEN wt cells with PTENi SF1670; $(n=7$ experiments; $n=40$ cells; $* \star \star ~ p=0.0002$. E) Aligned microfiber migration speed $(\mu \mathrm{m} / \mathrm{h})$ of HTB132 cells PTEN loss of function ( $n=3$ experiments; $n=37$ cells) and HTB26 PTEN wt cells $(\mathrm{n}=4 \text { experiments, } \mathrm{n}=35 \text { cells })^{* * * *} \mathrm{p}<0.0001$. 
A

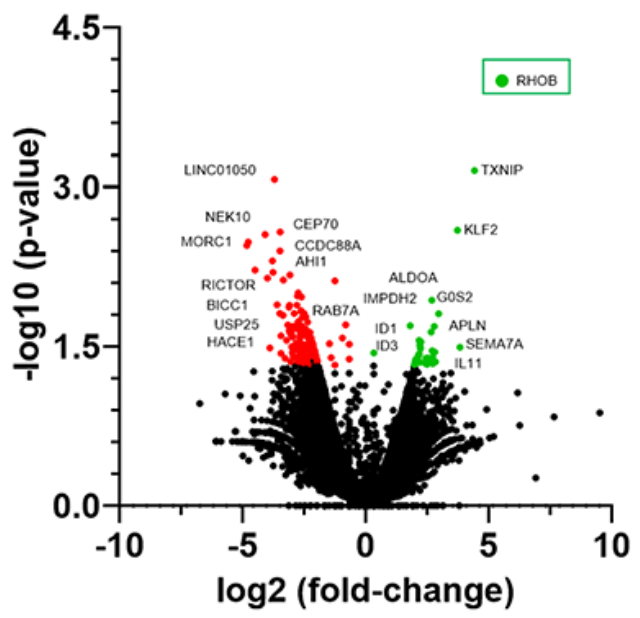

C

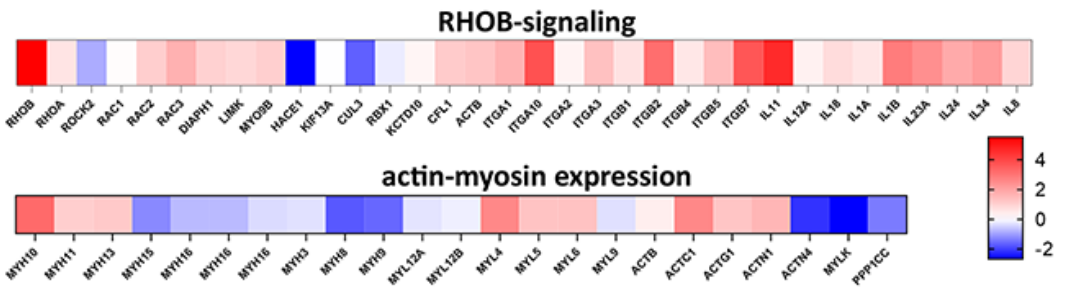

neuronal migration (nucleokinesis)

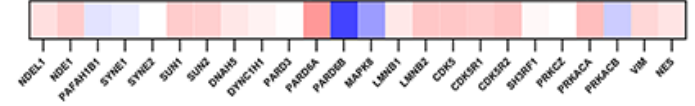

astrocyte migration (astrogliosis)

B
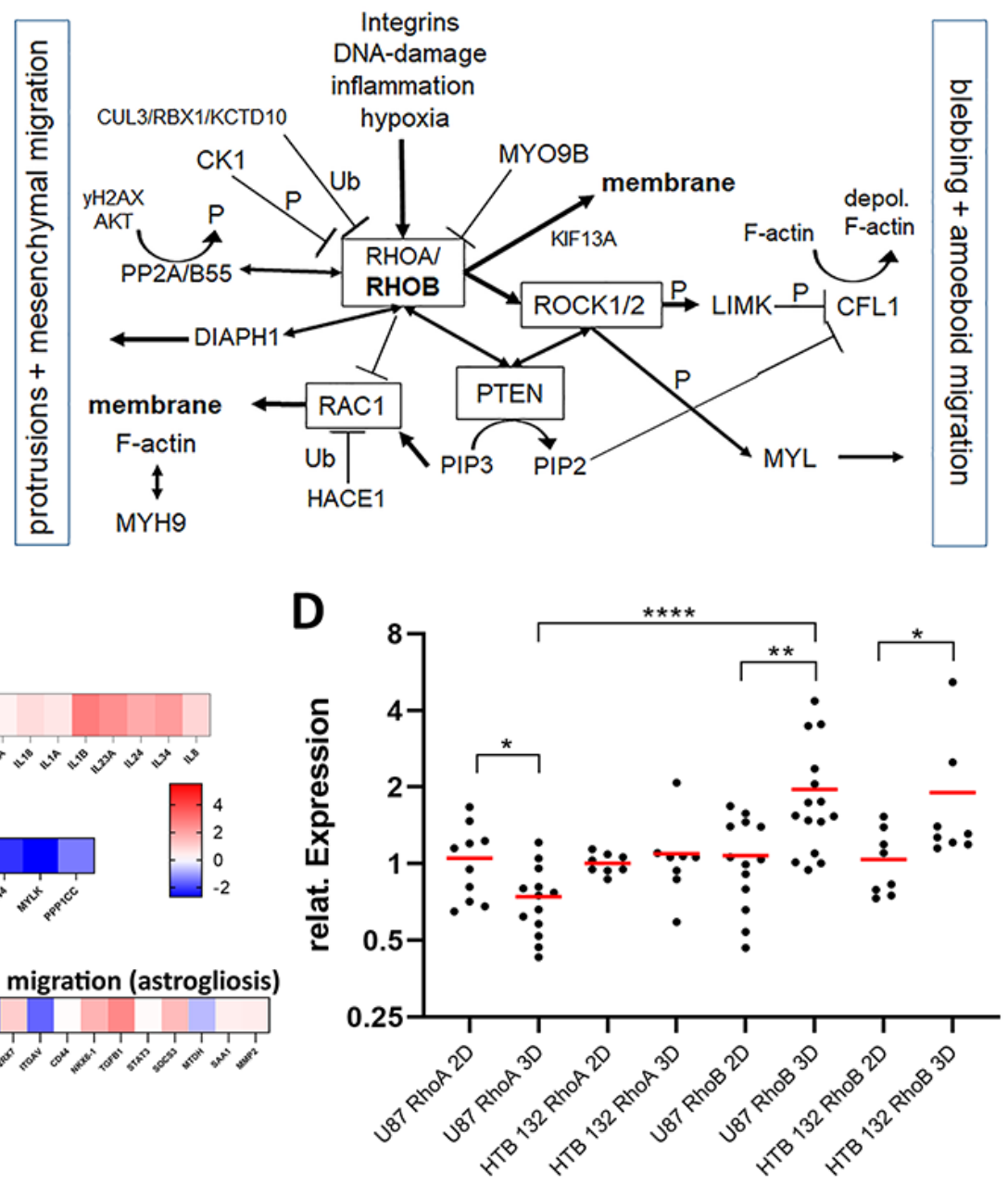

\section{Figure 5}

RHOB regulation of the migration pathway. A) RNA-seq data (Volcano-plot) of U87 GBM PTEN loss of function cells grown in 3D with Matrigel and scaffolds versus 2D (3 days). Red dots (left): significantly ( $p$ $<0.05)$ down regulated genes; green (right) dots significantly $(p<0.05)$ up-regulated genes. RHOB was 5.528 -fold log2 up-regulated in U87 GBM 3D cultures and scaffolds $(p=0.0001)$. Other significantly upregulated genes include G0S2 (6.4-fold, $p=0.011$ ), induced in glioma with high invasion [88], APLN (4.55fold, $p=0.0307$ ) induces colon cancer cell migration [89], ALDOA (3.37-fold, $p=0.0202)$ promotes lamellipodia [90], SEMA7A (3.84-fold, $p=0.05)$ via ITGB1 [91] and IMPDH2 (1.25-fold, $p=0.036$ ) regulates colorectal cancer cell migration [92] (Supplemental Tables 1, 2). B) RHOB signalling pathway overview. RAC1 promotes an elongated cell phenotype (mesenchymal) migration (left), which is inhibited by RHOB. RHO activates ROCK leading to blebbing and amoeboid migration (right), and negatively regulated by PTEN dephosphorylating PIP3 to PIP2. Ub (ubiquitinylated); P (phosphorylated). C) RNA-seq gene expression differences (Heatmaps) of U87 GBM PTEN loss of function cells in 3D with Matrigel and scaffolds versus U87 GBM in 2D (red=log2-fold up-regulated and blue=log2-fold down-regulated). Top heat map: RHOB signalling genes e.g. up-regulation of Integrins (esp. ITGA10 and ITGB2) and inflammation genes (e.g. IL11, IL1B) stimulating RHOB; Middle heatmap: actin-myosin expression; 
Bottom heatmap: neuronal migration (left) and astrocyte migration (right). D) qPCR and relative gene expression (2-aCt) of RHOA and RHOB in U87 GBM and HTB132 PTEN loss of function cells in 2D vs. U87 GBM and HTB132 cells in 3D with Matrigel and scaffolds (3 days). Significant values from left to right: U87 GBM RHOA expression in 2D vs. 3D: *p = 0.0316; U87 GBM RHOB expression in 2D vs. 3D: **p

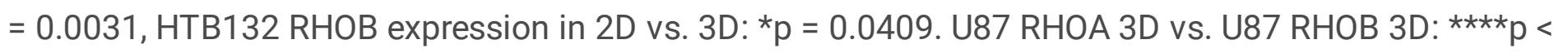
0.0001 .

A
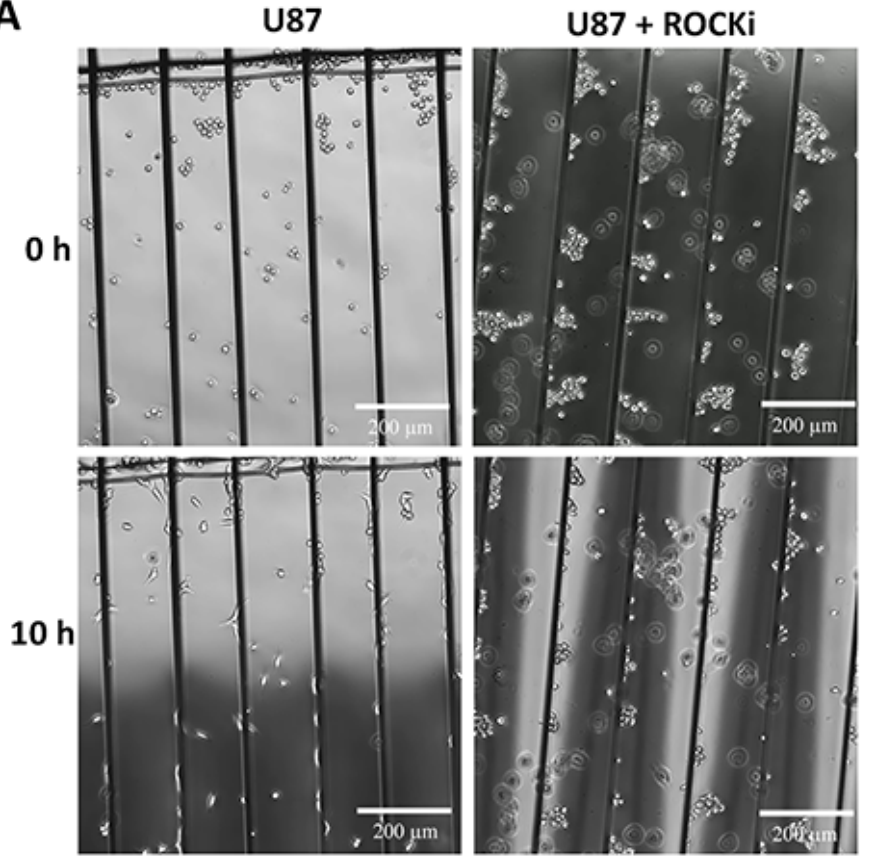

B

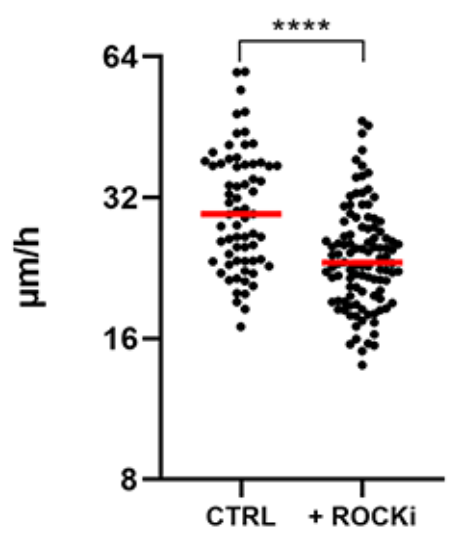

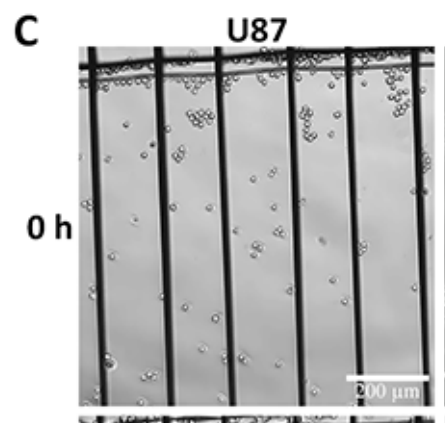
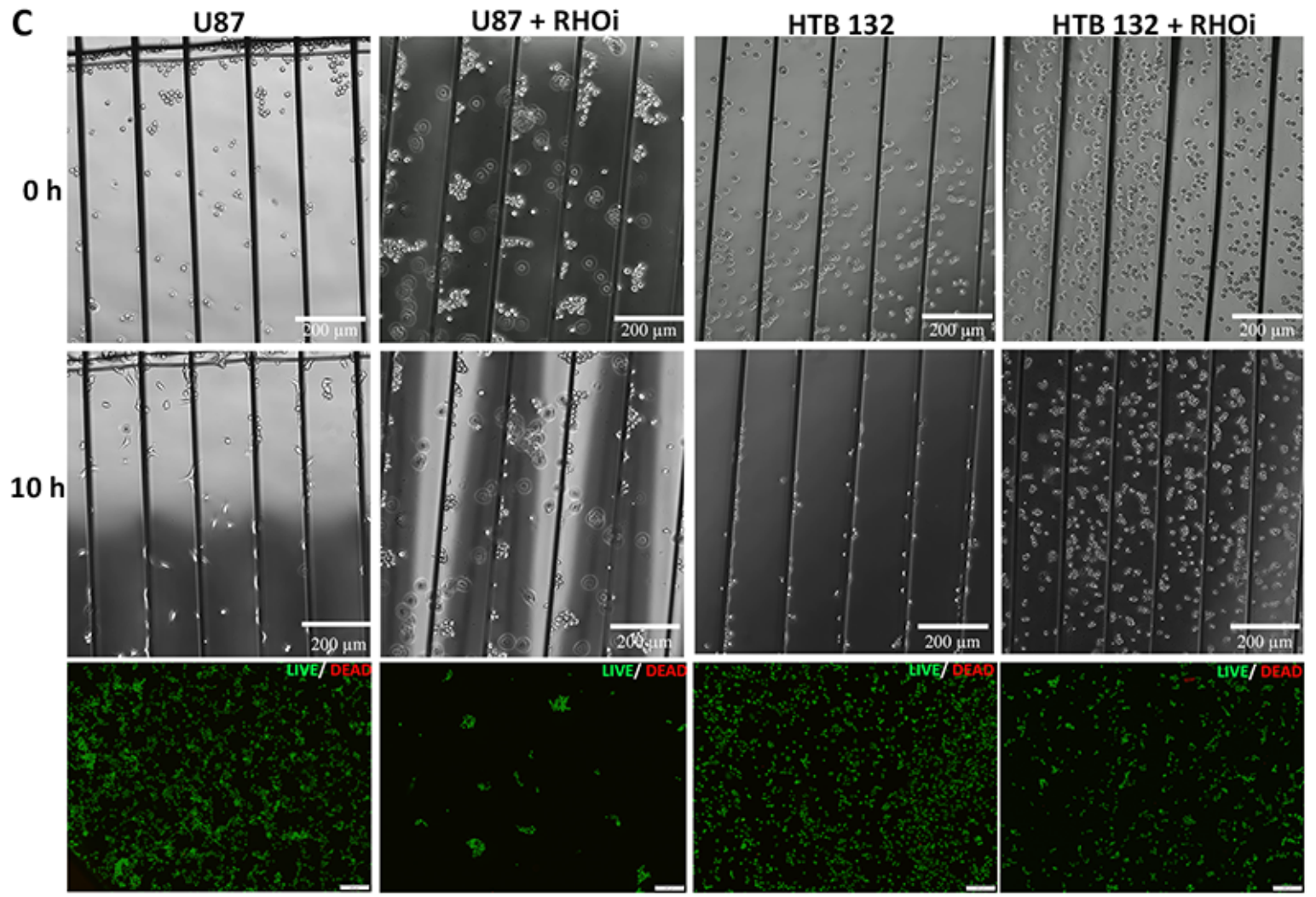

Figure 6 
RHO-ROCK regulates tumor cell durotaxis and migration. A) Representative images of U87 GBM PTEN loss of function control cells (left) or cells treated with the ROCKi Y-27632 at $0 \mathrm{~h}$ and $10 \mathrm{~h}$ on laminincoated aligned microfibers. B) Migration speed ( $\mu \mathrm{m} / \mathrm{h}$ ) of U87 GBM cells (control, CTRL) $(n=7$ experiments; $n=67$ cells) vs U87 GBM cells with the ROCKi Y-27632 ( $n=9$ experiments, $n=107$ cells); $\star \star \star \star ~ p<0.0002$. C) Representative images (from left to right) of U87 GBM control (1st column) or cells treated with RHOi (2nd column) and HTB132 PTEN wt cells control (3rd column) or treated with the RHOi (4th column) on aligned microfibers coated with laminin at $0 \mathrm{~h}$ and $10 \mathrm{~h}$ (U87: $\mathrm{n}=4$ experiments; HTB132: $n=3$ experiments). Below panel are examples of cell viability analyses of the respective tumor cells above after RHOi-treatment. Green: live cells; red: dead cells.

A
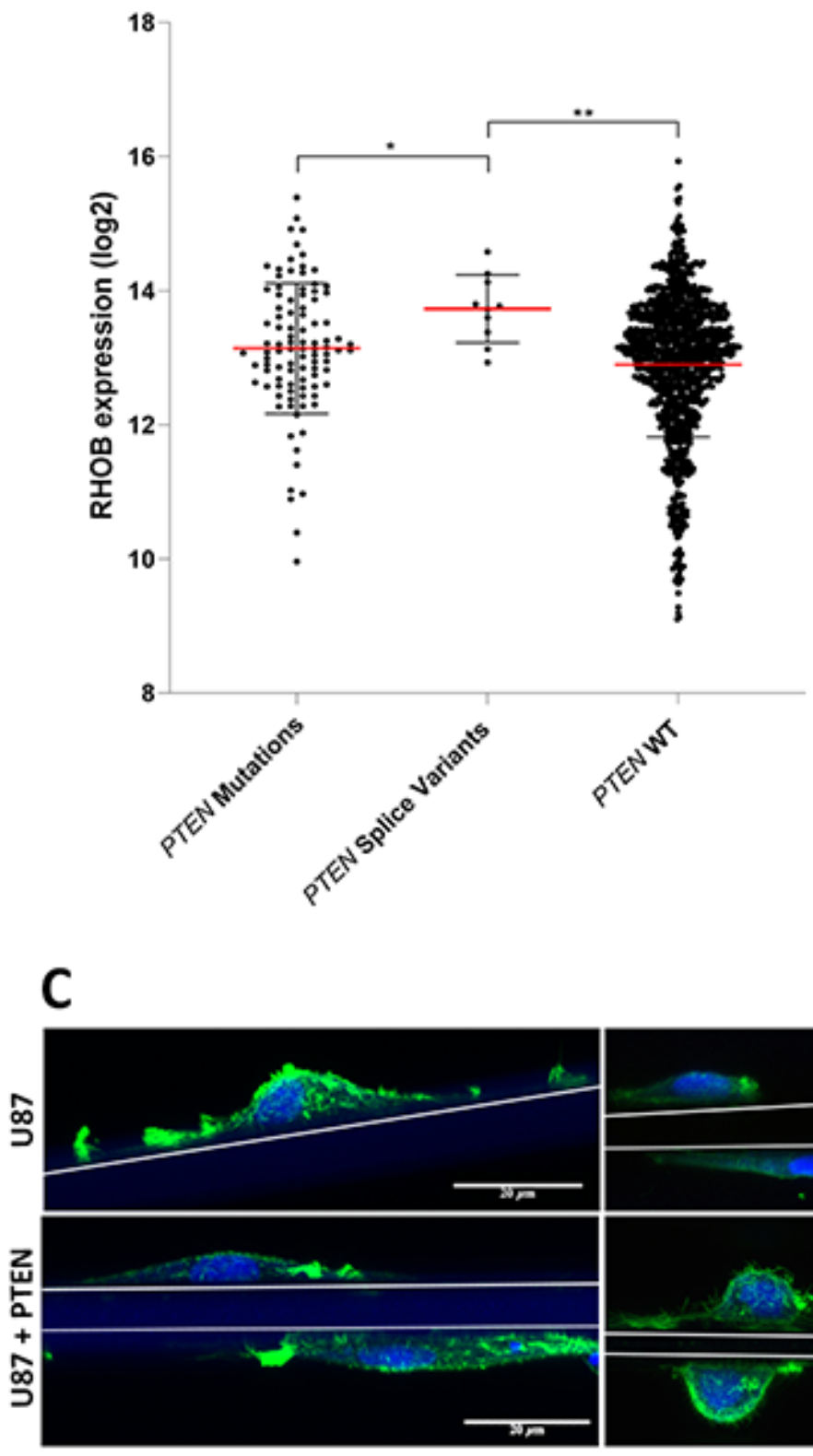

B

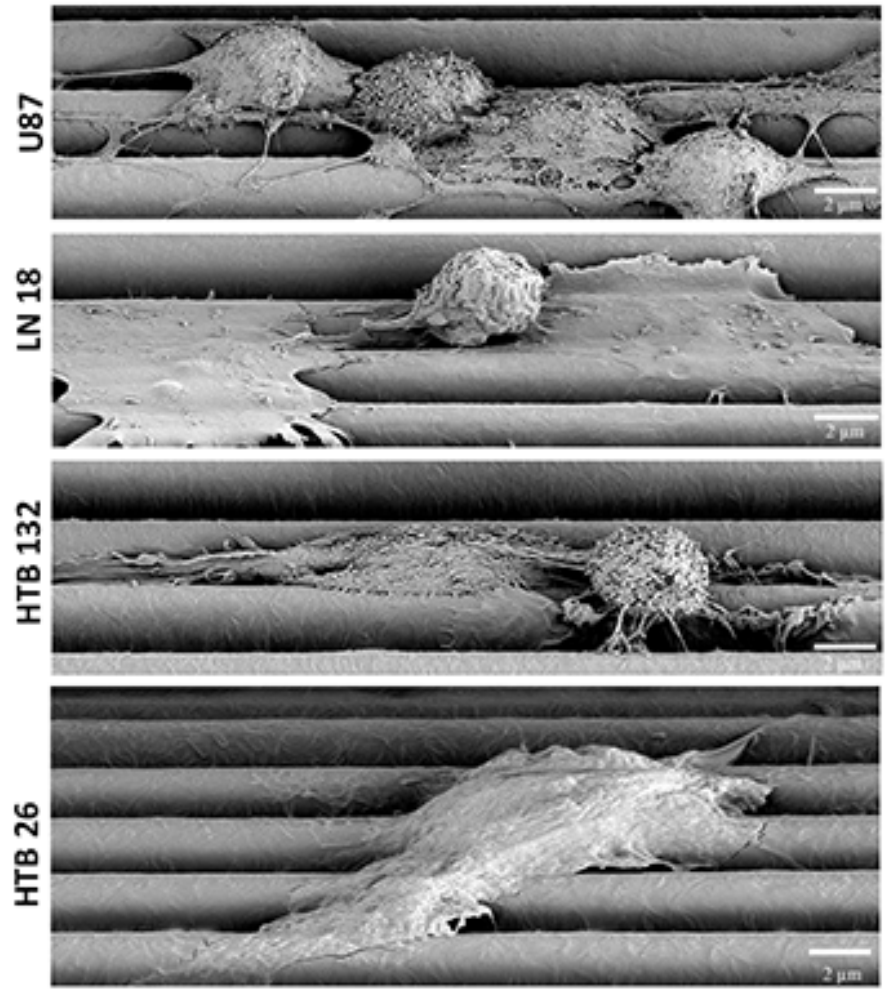

D

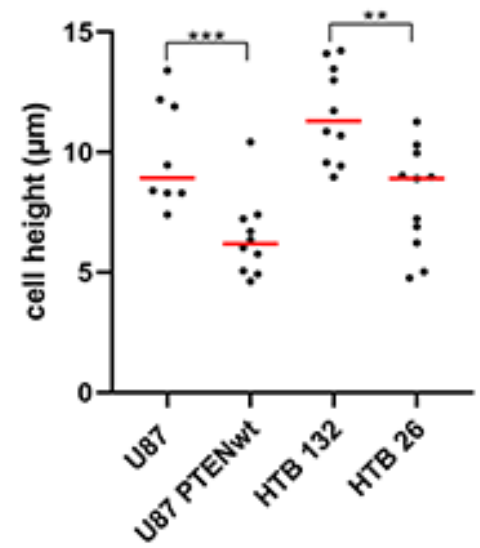

Figure 7 
RHOB signaling in primary GBM and breast tumors and $\mathrm{RHOB}$ regulation of cell morphology. A) Correlation of PTEN wt or PTEN splice or other mutations with RHOB expression from primary GBM and breast cancers (TCGA); ${ }^{*} p=0.031$; ${ }^{*} p=0.003$; PTEN mutations: $n=98$ tumors; including GBM $(n=46$ tumors) and breast cancer ( $n=52$ tumors). PTEN splice variants: $n=10$ tumors; including GBM $(n=4)$ and breast cancer $(n=6)$. PTEN wt: $n=1102$ tumors including GBM $(n=97)$ and breast cancer $(n=1005$ tumors). B) Representative SEM images on laminin-coated aligned microfibers with U87 GBM PTEN loss of function, LN18 GBM PTEN wt, HTB132 PTEN loss of function and HTB26 PTEN wt cells at $24 \mathrm{~h}$ after seeding (scale bar $=2 \mu \mathrm{m}$ ). C) Confocal z-stack images of F-actin Alexa 488 staining of U87 GBM PTEN loss of function control cells (top left) vs. U87 GBM cells after PTEN wt transfection and rescue (bottom left); and HTB26 PTEN wt (top) vs. HTB132 PTEN loss of function cells (bottom) on laminin-coated aligned microfibers at $24 \mathrm{~h}$ after seeding (green: F-actin; blue: DRAQ5 indicates nuclei) (scale bar $=20$ $\mu \mathrm{m})$. D) Quantification of cell height from confocal images $(\mu \mathrm{m})$ (Y-axis) of U87 GBM PTEN loss of function (control) vs. U87 GBM after PTEN wt transfection and rescue and HTB132 PTEN loss of function vs. HTB26 PTEN wt cells. ${ }^{\star \star \star} p=0.0010 ;{ }^{* \star} p=0.0016$. 

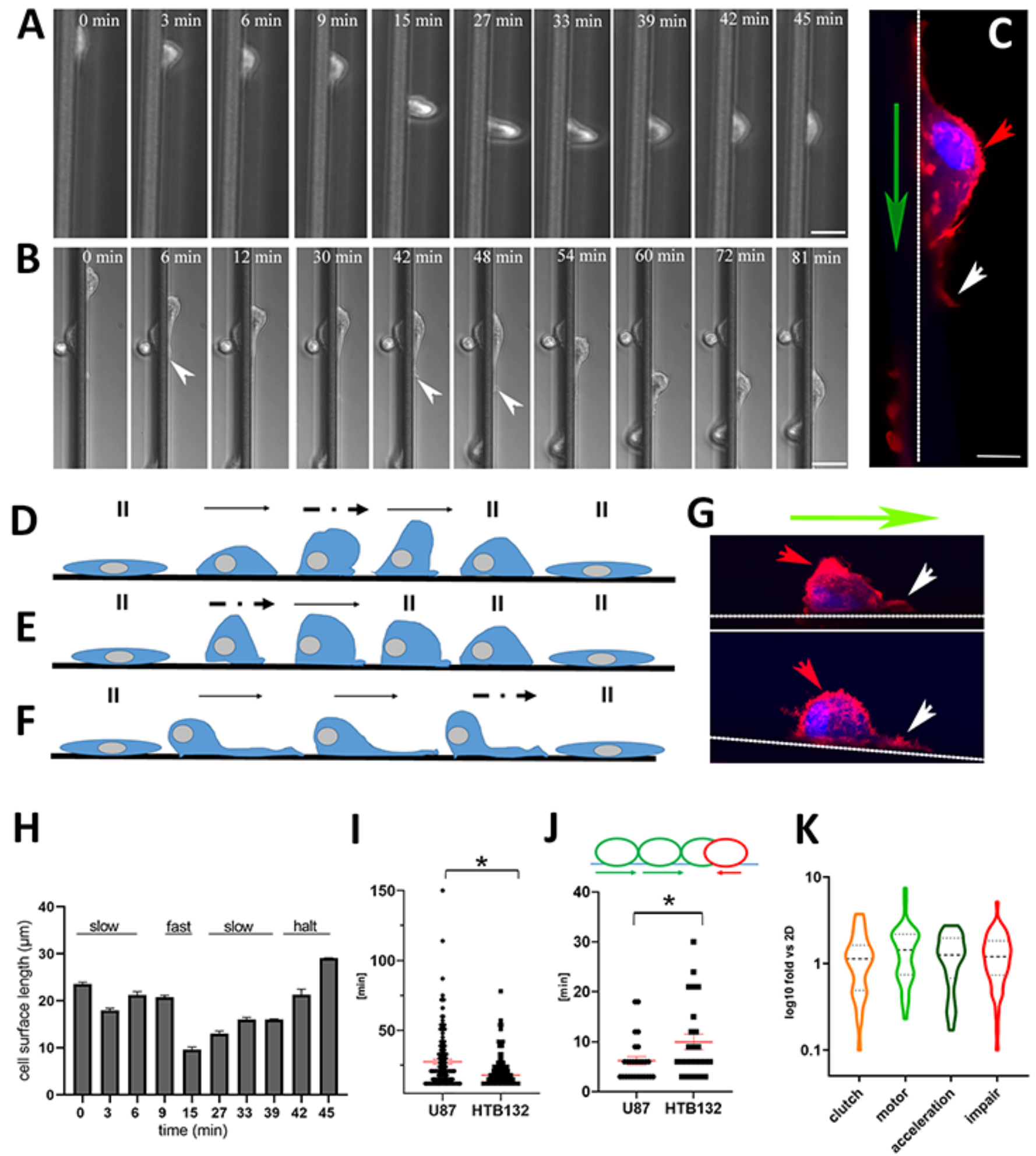

\section{Figure 8}

Skating snail-like cellular plasticity modes and persistence on aligned microfibers. A-B) Consecutive images of U87 GBM cell plasticity modes and migration over time; white arrows indicate leading edge cellular protrusions (scale bar $=25 \mu \mathrm{m}$ ). C) Confocal image of z-stacks showing F-actin Red 555 staining of an amoeboid U87 GBM PTEN loss of function cell (red: F-actin, blue DRAQ5 indicates nuclei), red arrow indicates cell nucleus at cell rear, white arrow indicates leading edge cellular protrusion, green arrow 
indicates direction of movement (scale bar $=10 \mu \mathrm{m}$ ). D-F) Schematic images of migration cell plasticity modes of "skating snail-like" movements for U87 GBM cells. II = non-moving resting cells; thin arrow = slow cell movement; bold dotted-thick arrow = fast cell movement $\mathrm{G}$ ) Confocal image of z-stacks of two U87 GBM cells (top, bottom) (red: F-actin, blue DRAQ5 indicates nuclei), red arrow indicates strong F-actin stain around the cell circumference, white arrow indicates cellular protrusion, and green arrow indicates direction of movement. H) Quantification of cell surface length on aligned microfibers ( $Y$-axis) of different U87 GBM migration modes (X-axis). Shortest bar graph = fastest mode of movement. I) Quantification of total U87 GBM and HTB132 cell migration time ( $\mathrm{min}$ ) for single cell movements along aligned microfibers, U87 GBM: $27.06 \pm 1.65 \mathrm{~min}$; HTB132: $21.08 \pm 1.09 \mathrm{~min} ;{ }^{*} \mathrm{p}=0.0142$. J) Quantification of reverse migration (min) of single movements of U87 GBM and HTB132 after continual forward movements (green circle and arrow = forward movement and red circle and arrow = reverse movement), U87 GBM: $6.22 \pm 0.83 \mathrm{~min}, \mathrm{HTB132}: 9.96 \pm 1.53 \mathrm{~min} ;{ }^{*} \mathrm{p}=0.0462$. A total of 258 single cell movements were analysed for both $8 \mathrm{I}$ and $8 \mathrm{~J}$. K) RNA-seq gene expression differences of U87 GBM cells in 3D with Matrigel and scaffolds versus U87 GBM in 2D. Expression of clutch genes $(n=35)$, motor genes $(n=40)$, acceleration genes $(n=50)$ and migration impairment genes $(n=58)[56-58]$.

\section{Supplementary Files}

This is a list of supplementary files associated with this preprint. Click to download.

- VideoS1U87dtfarnesylnoscaffold.mp4

- VideoS2U87dtfarnesylwithscaffold.mp4

- VideoS3U87alignmicrofiber.mp4

- VideoS4LN18alignmicrofiber.mp4

- VideoS5LN18withPTENi.mp4

- VideoS6HTB132alignmicrofiber.mp4

- VideoS7HTB26alignmicrofiber.mp4

- VideoS8U87withRHOi.mp4

- VideoS9U87skatingsnail.mp4

- VideoS10HTB132skatingsnail.mp4

- Wielandetal180821combined.pdf

- WielandSupplFigure1.tif

- WielandSupplFigure2.tif

- WielandSupplFigure3.tif

- WielandSuppleTable1.xlsx

- WielandSuppleTable2.xlsx 